\title{
De Sitter and Anti-de Sitter branes in self-tuning models
}

\author{
J.K. Ghosh, ${ }^{a}$ E. Kiritsis, ${ }^{b, a, 1}$ F. Nitti ${ }^{a}$ and L.T. Witkowski ${ }^{a}$ \\ ${ }^{a}$ APC, AstroParticule et Cosmologie, Université Paris Diderot, CNRS/IN2P3, CEA/IRFU, \\ Observatoire de Paris, Sorbonne Paris Cité, \\ 10, rue Alice Domon et Léonie Duquet, 75205 Paris Cedex 13, France \\ ${ }^{b}$ Crete Center for Theoretical Physics, Department of Physics, University of Crete, \\ 71003 Heraklion, Greece \\ E-mail: jghosh@apc.in2p3.fr, nitti@apc.in2p3.fr, lwitkow@apc.in2p3.fr
}

ABSTRACT: Maximally symmetric curved-brane solutions are studied in dilatonic braneworld models which realise the self-tuning of the effective four-dimensional cosmological constant. It is found that no vacua in which the brane has de Sitter or anti-de Sitter geometry exist, unless one modifies the near-boundary asymptotics of the bulk fields. In the holographic dual picture, this corresponds to coupling the UV CFT to a curved metric (possibly with a defect). Alternatively, the same may be achieved in a flat-space QFT with suitable variable scalar sources. With these ingredients, it is found that maximally symmetric, positive and negative curvature solutions with a stabilised brane position generically exist. The space of such solutions is studied in two different types of realisations of the self-tuning framework. In some regimes we observe a large hierarchy between the curvature on the brane and the boundary UV CFT curvature. This is a dynamical effect due to the self-stabilisation mechanism. This setup provides an alternative route to realising de Sitter space in string theory.

KEYwords: AdS-CFT Correspondence, Gauge-gravity correspondence

ARXIV EPRINT: 1807.09794

\footnotetext{
${ }^{1}$ http://hep.physics.uoc.gr/ kiritsis/.
} 


\section{Contents}

1 Introduction 1

2 A curved brane in a warped bulk $\quad 6$

2.1 (No) curved brane in a flat-sliced bulk 8

$\begin{array}{lll}2.2 & \text { Review of holographic RG flows for curved QFTs } & 10\end{array}$

$\begin{array}{lll}2.3 & \text { The junction conditions } & 17\end{array}$

2.4 Junction rules 20

2.5 Curved CFT boundary metrics vs. variable scalar sources 21

3 Solutions with IR fixed points $\quad 23$

3.1 IR-AdS with constant $U(\varphi) \quad 24$

3.2 IR-AdS with exponential $U(\varphi) \quad 33$

$4 \quad$ IR exponential potential $\quad 35$

4.1 Analytical results 36

$\begin{array}{lll}4.2 & \text { Numerical studies } & 37\end{array}$

5 Conclusions and outlook $\quad 41$

A Junction conditions for curved brane embeddings in a flat-sliced bulk $\quad 44$

A.1 Evanescent branes 46

B Perturbative analysis near the maximum of the potential $\quad 47$

$\begin{array}{ll}\text { C Regular IR geometries } & 49\end{array}$

D Perturbative solution of the junction conditions near a flat equilibrium $\begin{array}{ll}\text { point } & 51\end{array}$

\section{Introduction}

Soon after its first introduction in [1-3], it has been clear that the holographic gauge/gravity duality is intimately linked to a new way of thinking about modified gravity and beyond the standard model phenomenology which was being developed around the same time: the idea of the braneworld [4-6]. In these models, several problems of the Standard Model or its high-energy completion were addressed by postulating that the observed particles and fields are confined to a four-dimensional hypersurface (brane) embedded in a higher-dimensional space-time (bulk). The connection to holography, observed in [7-9], stems from the fact that the bulk was often taken to be (a portion of) Anti-de Sitter space, which may be 
given a dual interpretation in terms of a strongly coupled, large- $N$, four-dimensional field theory. Since then, holographic duality and braneworld phenomenology have often been two complementary sides of model building.

One of the earliest applications of braneworlds in this context was aimed at addressing the various naturalness problems which afflict the Standard Model and General Relativity, both from the particle physics side (electroweak hierarchy problem) and from the cosmology side (cosmological constant problem(s)).

On the one hand, cosmological applications led to departures from solutions describing a static flat brane in a static bulk, and prompted the study of braneworld cosmology [10-12]. The connection between braneworld models and gravity modifications was proposed as a way to model the observed current acceleration of the universe [13, 14].

On the other hand, it was proposed to use braneworld models to resolve the clash between the huge vacuum energy resulting from quantum effective field theory calculations and the smallness of the observed cosmological constant of the current de Sitter-like epoch. These proposal aimed at realising a self-tuning mechanism, first proposed in $[15,16]$, in which, contrary to purely four-dimensional models, the vacuum energy from quantum loops has no effect on the curvature of the brane, which is perceived as (almost) flat by fourdimensional observers $[17,18]$.

Although in principle appealing, the models which were proposed at the time all had issues related to the apparent inevitability of naked singularities in the bulk and/or an impossibility to have both a successful self-tuning mechanism and the existence of an effective four-dimensional gravity on the brane [19].

Recently, a novel framework was developed, which revisits the self-tuning braneworld approach [20] and which uses holography as a guiding principle for model building. It consists of a general two-derivative Einstein-dilaton bulk theory, and a codimension-one brane whose effective world-volume action contains all possible two-derivative terms (namely a brane potential for the scalar, an induced kinetic term for the scalar and an Einstein-Hilbert term for the induced metric) preserving four-dimensional diffeomorphism invariance. The bulk action is expected to be dual to a strongly coupled, large-N four-dimensional QFT, while the brane action is expected to contain the Standard Model fields as its localized fluctuations.

In the spirit of semi-holography (see e.g. [21]), asymptotically anti-de Sitter solutions of this theory are interpreted as a purely four-dimensional theory in which the bulk geometry is dual to a strongly interacting UV conformal field theory (CFT), deformed by a relevant operator (dual to the bulk scalar) and coupled to a weakly interacting Standard Model sector (the brane), a setup whose dual version was advocated in [22].

In the model described above, the brane separates the bulk geometry into two regions: one side connects to an asymptotically AdS conformal boundary (UV of the field theory dual). On the other side of the brane, the geometry may flow to another, regular, asymptotically AdS region (in which case the field theory flows to an IR conformal fixed point). ${ }^{1}$ The two sides of the geometry must obey the bulk Einstein-dilaton equations and

\footnotetext{
${ }^{1}$ It may also have a mild (resolvable) naked singularity, according to the Gubser criterion.
} 
the connection across the brane must satisfy Israel's junction conditions. Induced fourdimensional gravity on the brane is recovered in a range of distance scales via the DGP mechanism $[23,24]$ thanks to the localized Einstein-Hilbert term in the brane action. ${ }^{2}$

The use of holography as a guideline for model-building, in order to organise the space of solutions, has allowed to solve or alleviate some of the difficulties of the earlier models. In particular, holography can give a consistent meaning to certain kinds of bulk singularities [25]. These are indeed necessary to construct holographic duals of confining theories [26-28], and they may be consistently eliminated by uplifting to higher dimensions $[29,30]$.

As it was shown in [20], for rather generic choices of the bulk and brane potentials, enforcing the holographic interpretation of the model results in a self-tuning mechanism for the four-dimensional cosmological constant. The model admits solutions in which the geometry on the brane is flat, regardless of the vacuum energy arising from quantum loops of the brane fields. The brane is stabilized in the bulk at an equilibrium position, which is dynamically determined by the bulk geometry and brane potentials via Israel's junction conditions. Under certain general conditions, all fluctuations around the equilibrium position have positive energy.

The fact that the framework proposed in [20] allows self-tuning flat solutions opens new questions, and at the same time offers new possibilities for model building. In [20], brane flatness and four-dimensional Poincaré invariance were imposed by design on the solution ansatz, and the self-tuning mechanism corresponds to the existence of stabilized solutions with this symmetry. It is important however to explore, in the same context, other solutions in which the brane has non-zero curvature and/or has a time-dependent (cosmological) induced metric. One reason is to understand how these solutions compete with the flat solution (which represents the Poincaré-invariant vacuum). In addition, because we currently live in an accelerating universe, obtaining a positively curved (e.g. de Sitter) metric on a brane is phenomenologically important. Finally, it is important to clarify what is responsible, from the dual field theory perspective, for obtaining a curved brane geometry. Exploring these questions is the purpose of the present work.

In this paper, we look for solutions of the self-tuning framework in which the brane has a curved geometry. Our first result can be formulated as follows: no vacuum curved-brane solutions generically exist. ${ }^{3}$ A non-trivial brane geometry can be obtained if one modifies the UV boundary conditions on the bulk fields, such that they allow domain wall solutions with constant curvature radial slices. In the dual QFT language this amounts to changing the dual QFT. As we shall see, a de Sitter brane geometry will be possible when the domain wall solution is sliced by de Sitter slices. In this case, the bulk geometry is dual to a QFT defined on a constant positive curvature manifold. For an AdS brane geometry, the bulk geometry is a domain wall with negative curvature slices, and the UV field theory is a CFT with an additional defect $[31,32]$.

\footnotetext{
${ }^{2}$ This term is generated via quantum effects of the brane localized fields.

${ }^{3}$ By "vacuum" solution here we mean one where the bulk has 4d Poincaré invariance, representing the ground state of the dual QFT.
} 


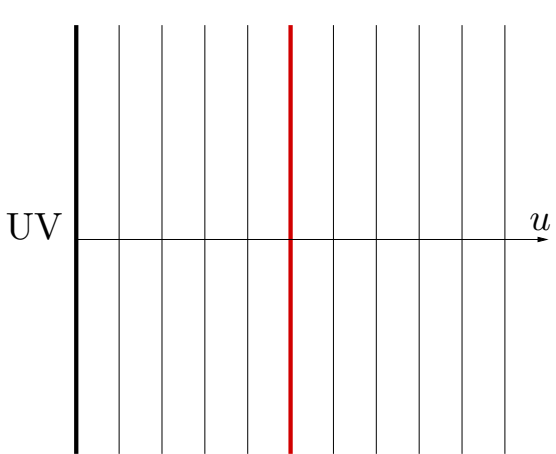

(a)

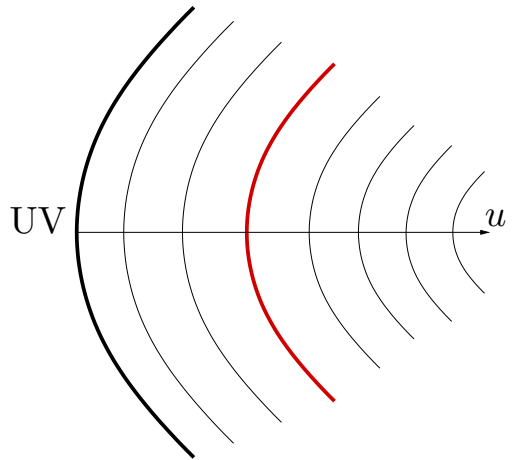

(b)

Figure 1. A sketch of the solutions allowing for a flat brane (a) and those allowing for a curved brane (b). The thick black line represents the conformal boundary of AdS, whereas the brane is represented in red. The direction $u$ is the holographic direction, and the thin black lines are constant- $u$ hypersurfaces. The brane metric is inherited from the UV boundary metric (up to a rescaling).

Prompted by the above result, we set forward to study curved domain wall geometries, and we ask the question whether stabilized curved brane solutions do arise. We focus in particular on maximally symmetric geometries, in which both the bulk and the brane preserve four-dimensional de Sitter (dS) or Anti-de Sitter (AdS) invariance. The structure of these solutions is sketched in figure 1, in which we show a comparison between the flat solutions studied in [20] and the curved embeddings we discuss in this paper.

As far as the bulk is concerned, the corresponding solutions describe holographic RG flows on maximally symmetric spaces, and they were studied extensively in [33], to which the reader is referred for details. The introduction of the brane amounts to gluing together two bulk RG-flow solutions of the type described in [33], to impose regularity on the IR side of the solution and to solve at the same time for the brane position and the UV part of the geometry in such a way that Israel's junction conditions are satisfied.

In these solutions, the metric on the brane is the same (up to a scaling factor, which depends on the position in the bulk) as the UV metric to which the dual CFT is coupled. For example, a de Sitter brane solution can exist only if the dual CFT is set on de Sitter space, and similarly for Anti-de Sitter. In the latter case, the holographic interpretation is more subtle because, in addition to the usual boundary conditions in the radial direction, one has also to introduce boundary conditions at the boundary of the slices. As discussed in [33] (see also [31] for a previous similar discussion) this introduces a codimension-one defect in the dual field theory.

Although the type of geometry of the brane is fixed by the boundary conditions, the magnitude of the brane curvature and its position in the bulk are determined dynamically by the field equations and the junction conditions. We refer to this as self-stabilisation, which is the curved counterpart of the self-tuning mechanism found in [20]. ${ }^{4}$

\footnotetext{
${ }^{4}$ We reserve the phrase self-tuning for the flat solutions, in which the effective cosmological constant on the brane is dynamically set to zero.
} 
There is an alternative realisation of the same solutions, which can be obtained via a bulk coordinate transformation, in which the leading UV asymptotics correspond instead to a flat metric, but the scalar sources are varying in space or time. This leads to an inequivalent description in terms of the dual QFT: instead of a boundary QFT living on a curved space-time, we have a flat-space QFT driven by a time-varying (in the dS case) or space-dependent (in the AdS case) source. The two descriptions however result in the same brane geometry. Although in the bulk the two solutions are related by a coordinate transformation, the latter acts non-trivially on the boundary, and it leads therefore to an inequivalent theory with different boundary sources. In most of the paper we will work with the curved-CFT description with constant scalar sources.

The solutions we study are extensions to general dilatonic braneworld models, with general induced gravity and kinetic terms, of the curved braneworld solutions first introduced by Kaloper in [34] and by Karch and Randall in [35], in which the embedding of a curved brane whose tension was de-tuned from the RS [6] value was first studied. Further generalisation, which considered asymmetric setups and/or included an induced Einstein-Hilbert term, were explored e.g. in [36-40] and recently revisited in [41]. The main difference between those models and the ones studied here is the effect of self-stabilisation: both the bulk solution and the brane geometry are determined dynamically, by the interplay between the running of the bulk scalar and the brane potential and kinetic terms. As a consequence, any hierarchy which is produced between the CFT curvature (the UV boundary conditions) and the brane curvature has a dynamical origin. Also, in previous works, the two sides of the bulk geometry were often taken to be pure AdS space-times with different curvatures. In our case, instead, both sides of the geometry are different solutions of the same bulk theory. This allows for a consistent holographic interpretation. Finally, the presence of the scalar field and the self-stabilisation mechanism are important for perturbative stability, which was shown to be a problem for the pure-gravity models [40, 42].

After a general discussion, we focus on two specific models, which were those presented in [20] as concrete realisations of the self-tuning mechanism. They share a similar UV structure but differ in the IR, as the first one admits a regular IR fixed point where the dilaton reaches a finite value, while the second one has a "good" IR singularity where the dilaton diverges. Although the former model has the nice property of having a regular interior, it is the latter which is more promising for phenomenology, as it is more suitable to implement the self-tuning mechanism without violating the conditions for stability of the flat equilibrium solution. In the curved case we study here, the difference between these IR geometries gives rise to different phenomenology of the stabilisation mechanism, and the corresponding scaling relations between brane, bulk, and boundary curvatures.

The phenomenology of self-stabilisation in the various examples may differ from case to case, though a few features are worth mentioning. Generically, we find that one or more solutions exist for both positive and negative curvature, with one branch always connected to the flat self-tuning solution. Along this branch, the brane curvature can be continuously tuned to zero by decreasing the value of the UV curvature. Interestingly, we find situations where the brane curvature is bounded by a maximum value, no matter how large one takes the boundary curvature. 
From the phenomenological perspective, our approach is relevant to the general discussion of how to find de Sitter (or more generally, accelerating) solutions in string theory, which recently has received renewed attention. The reason is that, as argued first in [43] the braneworld setups described here can arise in warped compactifications of string theory. On the one hand, traditional methods based on compactifications from ten dimensions, with various combinations of brane and fluxes, have a hard time realizing dS space, [44, 45]. On the other hand, it has been recently argued that de Sitter solutions are forbidden in a quantum gravity theory by generalisations of the weak gravity conjecture [46]. The class of solutions we find here in the context of holography offer an alternative way of producing de Sitter, on the brane, compared to methods based on engineering bulk solutions, as was recently advocated in [44]. In this sense our results agree with the general arguments that de Sitter cannot arise as a vacuum solution: rather, as it is found here, it must be driven by a non-trivial UV coupling of a CFT to curved metric sources.

As a final remark, we note that more general curved-brane solutions in which, in the bulk, both the scale factor and the dilaton have a non-trivial time-dependence are expected to exist, even without departing from UV asymptotics corresponding to a flat UV CFT metric. These solutions however are expected to describe a cosmological, non-vacuum state. Generically, these will not describe exact de Sitter space, although in some regions of parameter space they may have a quasi-de Sitter regime (similar to slow-roll inflation or quintessence). Their study is needed to answer the important question regarding timeevolution (and approach to equilibrium) and cosmology of the self-tuning model. This will be the subject of future work [47].

This paper is organized as follows. In section 2 we introduce the setup, present the general results, review the bulk geometries corresponding to curved holographic RG flows, discuss the junction conditions for curved branes, and the connection with the flat CFT description. The rest of the paper is devoted to exploring the space of constant positive or negative curvature solutions in specific models, and the results are mostly obtained numerically. In section 3 we look for curved brane solutions in a model with a conformal IR fixed point. In section 4 we turn to models with an asymptotically exponential dilaton potential, and study solutions in which the brane is stabilized at a large value of the dilaton field. Several technical details are left to the appendices.

\section{A curved brane in a warped bulk}

We will consider Einstein-scalar theory in $d+1$ dimensions, coupled to a $d$-dimensional dynamical hypersurface (brane). The bulk space-time is parametrized by coordinates $\left(u, x^{\mu}\right)$ and we consider both the Euclidean and Lorentzian metric, for which we take the signature $(-,+,+, \cdots,+)$ for the $x^{\mu}$ coordinates. We will work with the most general two derivative action for this set-up:

$$
S=S_{\text {bulk }}+S_{\text {brane }},
$$


where

$$
\begin{aligned}
S_{\text {bulk }}[g, \varphi] & =M_{P}^{d-1} \int d u d^{d} x \sqrt{-g}\left(R^{(g)}-\frac{1}{2} \partial_{A} \varphi \partial^{A} \varphi-V(\varphi)\right)+S_{\mathrm{GHY}}+S_{\mathrm{ct}}, \\
S_{\text {brane }} & =M_{P}^{d-1} \int d^{d} x \sqrt{-\gamma}\left(-W_{B}(\varphi)-\frac{1}{2} Z(\varphi) \gamma^{\mu \nu} \partial_{\mu} \varphi \partial_{\nu} \varphi+U(\varphi) R_{B}\right) .
\end{aligned}
$$

Here, $g_{A B}$ is the bulk metric, $R^{(g)}$ the bulk Ricci scalar, $\gamma_{\mu \nu}$ the induced metric on the brane, $R_{B}$ the corresponding Ricci scalar, $V(\varphi)$ is the bulk potential, $S_{\mathrm{GHY}}$ is the GibbonsHawking-York term, and $S_{\text {ct }}$ a boundary counterterm action needed for holographic renormalisation, and whose details here are unimportant. The bulk solution and the brane embedding in the bulk are determined by the bulk Einstein's equations and by imposing Israel's junction conditions across the co-dimension-one brane.

The framework above was considered in [20] as a way to describe, in a gravity dual language, the interaction between weakly coupled physics (e.g. the Standard Model) localized on the brane, and a strongly coupled, large $N$ CFT, described by the bulk geometry. In this context, the functions $W_{B}(\varphi), Z(\varphi)$ and $U(\varphi)$ may be thought as generated by integrating out the brane-localized fields. In particular the function $W_{B}(\varphi)$ contains contributions from the brane vacuum energy.

It was shown in [20] that, rather generically, this kind of models allow self-tuning solutions, in which the brane geometry is flat, regardless of the value of the brane vacuum energy. In that work, the bulk geometry enjoyed four-dimensional Poincaré invariance of constant- $u$ hypersurfaces, which was inherited by the brane.

In this work, we will move beyond flat brane solutions, and ask the question, what kind of non-trivial brane geometries one can obtain within the same framework. After some general considerations, we will then restrict our attention to constant curvature brane geometries, i.e. either de Sitter or Anti-de Sitter. We will not study the most general solution of the model specified by the action (2.2)-(2.3), but we will restrict to situations in which the bulk is static, leaving more general time-dependent geometries for future work.

The simplest possibility to move in this direction is to look for a solution in which the curvature is due solely to the embedding of the brane, and the bulk geometry retains its four-dimensional Poincaré invariance. In this ansatz, the boundary conditions (which define the dual CFT data) are the same ${ }^{5}$ as those studied in [20]. As we will show in section 2.1 however, for generic bulk and brane potentials, no solution of this kind exists. This leads us to generalize the bulk ansatz, in a way described in section 2.2.

In order to find a curved brane embedding in a static bulk we will need to modify the metric asymptotics. In the dual CFT language this means that we have to couple the boundary field theory to a non-trivial metric $\zeta_{\mu \nu}^{\mathrm{UV}}$. In the gravity dual, the simplest ansatz describing this situation while keeping the solution static ${ }^{6}$ takes the form

$$
\varphi=\varphi(u), \quad d s^{2}=d u^{2}+e^{2 A(u)} \zeta_{\mu \nu} d x^{\mu} d x^{\nu}
$$

\footnotetext{
${ }^{5}$ In the dual CFT language such a solution would constitute an alternative state of the same theory which gave the self-tuning vacuum.

${ }^{6}$ In a sense which we will specify more precisely below.
} 
where $A(u)$ is the warp factor and $\zeta_{\mu \nu}$ a fiducial $d$-dimensional metric. The ansatz (2.4) is the simplest static solution such that a brane embedded at a fixed $u$ has a non-trivial world-volume curvature. Even though the metric $\zeta_{\mu \nu}$ may depend explicitly on $t$ (e.g. it may be a $d$-dimensional FRW metric), we still call this metric static because the functions to be solved for (namely $A(u)$ and $\varphi(u)$ ) depend only on the holographic coordinates and not on time. More complicated bulk solutions are possible, and we will comment briefly on them.

We will assume that the bulk has an asymptotic near-boundary region (which we can choose to be reached as $u \rightarrow-\infty$ ) where the solution takes the form of a Fefferman-Graham expansion, in which the leading term defines the boundary QFT metric $\zeta_{\mu \nu}^{\mathrm{UV}}$,

$$
d s^{2} \simeq d u^{2}+e^{-\frac{2 u}{\ell}}\left[\zeta_{\mu \nu}^{\mathrm{UV}}+\ldots\right] d x^{\mu} d x^{\nu}, \quad u \rightarrow-\infty
$$

If $\zeta_{\mu \nu}^{\mathrm{UV}}=\eta_{\mu \nu}$, the equation above is the leading near-boundary behavior of the metric of the Poincaré patch of $(d+1)$-dimensional Anti-de Sitter space. For $(2.5)$ to hold, it is enough that the bulk potential has a local maximum (say at $\varphi=0$ ) where it takes on a negative value, $V(0)=-d(d-1) / \ell^{2}$. We will give a more detailed description of both the near-boundary (UV) and the interior (IR) regions in section 2.2.

By adjusting a free additive constant in $A(u)$ we can always identify the fiducial slice metric $\zeta_{\mu \nu}$ in (2.4) with the metric $\zeta_{\mu \nu}^{\mathrm{UV}}$ in $(2.5)$. Therefore from now on we will assume

$$
\zeta_{\mu \nu}=\zeta_{\mu \nu}^{\mathrm{UV}} .
$$

In general, a co-dimension-one brane configuration preserving space-rotations is described by an embedding of the form ${ }^{7} F(u, \tau)=0$ for some function $F$, or more explicitly by giving a trajectory in the holographic direction,

$$
u=u_{\star}(\tau) .
$$

The induced metric on the brane takes the form

$$
d s^{2}=\left[\left(\frac{d u_{\star}}{d \tau}\right)^{2}+e^{2 A\left(u_{\star}(\tau)\right)} \zeta_{\tau \tau}\right] d \tau^{2}+e^{2 A\left(u_{\star}(\tau)\right)} \zeta_{i j} d x^{i} d x^{j} .
$$

The situation considered in [20] was the case of a static, flat brane located at $u=u_{\star}$ and separating two different geometries (one for $u<u_{\star}$, one for $u>u_{\star}$ ) of the form (2.4) with flat slices, $\zeta_{\mu \nu}=\eta_{\mu \nu}$, and different scale factors. Here, we want to look at more general solutions which allow for a curved brane.

\section{1 (No) curved brane in a flat-sliced bulk}

First, we address the question whether a constant curvature brane can be embedded in a flat-sliced bulk, i.e. we take the bulk geometry to be the same as in [20],

$$
d s^{2}=d u^{2}+e^{2 A(u)} \eta_{\mu \nu} d x^{\mu} d x^{\nu}, \quad \varphi=\varphi(u)
$$

\footnotetext{
${ }^{7}$ For definiteness, here we focus on a "cosmological" brane, whose induced metric depends non-trivially on the time-coordinate $\tau$. Similar considerations apply a static curved brane, after trading $\tau$ for one of the space coordinates.
} 
but look for a more general brane embedding, specified by a non-trivial function $u_{\star}(\tau)$. Such a solution would result in a curved "cosmological" brane. The bulk scale factor and scalar field on each side of the brane are a priori different solutions of the the bulk Einstein's equation,

$$
(A, \varphi)= \begin{cases}\left(A_{-}(u), \varphi_{-}(u)\right) & u<u_{\star}(\tau) \\ \left(A_{+}(u), \varphi_{+}(u)\right) & u>u_{\star}(\tau)\end{cases}
$$

Israel's junction conditions then dictate how the left and right solutions must be glued across the brane. The question is whether, for a given bulk theory and a given choice of brane potentials, it is possible to find a non-trivial embedding function such that Israel's junction conditions are satisfied.

In general, the answer is negative: as we show in detail in appendix A, for generic choices of the brane potentials, no solutions to the junction conditions may be found: for a non-trivial embedding function $u_{\star}(\tau)$ the junction conditions require that all world-volume terms in the brane action (2.3) must vanish. ${ }^{8}$ As we discuss in appendix A, for special choices of the brane potentials however, non-trivial solutions may be found. These have the curious property that, although these solutions are exact, there is no backreaction of the brane onto the bulk (evanescent branes). For example, if $W_{B}, U$ and $Z$ are positive constants, then a de Sitter embedding of the brane exists if the bulk is Poincaré-AdS space-time with constant scalar field. More generally, it may be possible to tune the brane potentials so that a certain FWR embedding is possible in a given bulk solution. These special cases however require the brane potentials to be tuned to specific functions so that the corresponding brane embedding is compatible with the bulk solution. Although this is be interesting, this goes against our general philosophy, which consists in taking the bulk and brane data as unrelated and as generic as possible.

Given the result above, we have two possibilities for obtaining a curved brane embedding:

1. We can keep a flat UV metric $\zeta_{\mu \nu}^{\mathrm{UV}}=\eta_{\mu \nu}$ but generalise the bulk ansatz (2.4), embedding a non-trivial brane trajectory $u_{\star}(\tau)$ in a time-dependent bulk of the general form

$$
d s^{2}=n^{2}(u, \tau) d u^{2}+\beta^{2}(u, \tau) d \tau^{2}+\gamma^{2}(u, \tau) \delta_{i j} d x^{i} x^{j}
$$

We can then impose boundary conditions such that the metric reduces asymptotically to the form (2.5) with $\zeta_{\mu \nu}^{\mathrm{UV}}=\eta_{\mu \nu}$, with time-dependent corrections entering only at subleading orders.

2. Alternatively, we can study solutions in which the brane is static, and at a fixed position $u=u_{\star}$, in a static bulk solution like (2.4) where each slice is curved. The induced metric on the brane will inherit the curvature of the corresponding constant$u$ slice. This choice necessarily leads to a near-boundary expansion of the metric like

\footnotetext{
${ }^{8}$ This may still lead to interesting physics if we can treat the brane as a probe, as is the case in the so called mirage cosmology $[48,49]$. In this case the trajectory $u_{\star}(t)$ is determined by extremizing the world-volume action in a fixed background, ignoring the backreaction on the bulk. However this is not our goal here, as we want to keep the backreaction intact. It will be studied in another publication.
} 
in (2.5), with a curved UV metric, which amounts to couple the dual UV CFT to a curved background. ${ }^{9}$ Note that this involves different AdS boundary conditions and is therefore not in the same class of solutions as asymptotically flat conditions. However, as we will see in subsection 2.5 , it is possible to rewrite these solutions in terms of a flat space CFT, which however is coupled to time- or space-dependent external sources.

The first choice, appropriate for the important question of the the cosmological evolution of the self-tuning model, is not the road we will pursue here, and we it will be analysed in a separate paper, [47].

In the rest of this paper we will explore the second option, and embed the brane as a static hypersurface in a bulk metric of the form (2.4), where $\zeta_{\mu \nu}$ is identified with the metric of the dual UV CFT. However these solutions are not unrelated to the first option described above: as we will discuss in more detail in section 2.5, a coordinate transformation can bring a solution of the form (2.4) to one of the form (2.11) with flat asymptotic conditions, at the cost of introducing a time- or space-dependence in the scalar field at leading order in the near-boundary expansion. In the holographic dual language, this situation describes a CFT living on flat space, but driven by a varying scalar source.

\subsection{Review of holographic RG flows for curved QFTs}

From now on we focus on a setup in which the brane is the interface between two different geometries of the type (2.4), each one characterized by a warp factor $A(u)$ and a scalar field profile $\varphi(u)$. One of them connects with the boundary of AdS (UV), the other should have a regular interior (IR).

The induced metric on the brane is, up to a constant scaling, the same as the slice metric (and the UV metric),

$$
\gamma_{\mu \nu}=e^{2 A\left(u_{\star}\right)} \zeta_{\mu \nu}
$$

and the induced curvature scalar is

$$
R_{B}=e^{-2 A\left(u_{\star}\right)} R^{(\zeta)} .
$$

Because we are working with $\zeta_{\mu \nu}=\zeta_{\mu \nu}^{\mathrm{UV}}$, the brane geometry is the same (up to an overall scaling) as the UV metric of the space where the CFT lives.

For simplicity, from now on we will restrict to bulk geometries (2.4) whose constant$u$ slices are maximally symmetric d-dimensional hypersurfaces, whose metric $\zeta_{\mu \nu}$ has the property

$$
R_{\mu \nu}^{(\zeta)}=\kappa \zeta_{\mu \nu}, \quad R^{(\zeta)}=d \kappa .
$$

\footnotetext{
${ }^{9}$ The same considerations applies to a more general class of bulk metrics than (2.4), of the form

$$
d s^{2}=d u^{2}-\beta^{2}(u) d \tau^{2}+a^{2}(\tau) \gamma_{i j}(u) d x^{i} d x^{j} .
$$

These metrics have the property that any surface at $u=u_{\star}$ has a fixed FRW geometry, which can be brought in standard form after a world-volume coordinate transformation acting on $\left(\tau, x_{i}\right)$. However different constant- $u$ slices differ by more than just an overall rescaling. One can easily show that, also in this case, the near-boundary expansion leads to a non-trivial time-dependent UV metric.
} 
The constant $\kappa$ is given by

$$
\kappa=\left\{\begin{array}{cl}
\frac{(d-1)}{\alpha^{2}} & \mathrm{dS}_{d} \\
0 & \mathcal{M}^{d} \\
-\frac{(d-1)}{\alpha^{2}} & \mathrm{AdS}_{d}
\end{array},\right.
$$

where $\alpha$ is the radius of curvature. ${ }^{10}$ Whether $\kappa$ is positive, negative or zero is fixed by the UV boundary conditions, i.e. by the metric to which the CFT is coupled.

One of the reasons we restrict to maximally symmetric slices is practical: in this case the bulk geometry was studied in detail in [33] and we can use the results of that work. However, the case of constant positive curvature is already interesting for phenomenology, as it can relate both to primordial inflation and to late time cosmology, both described by quasi-de Sitter space-time. Extensions to a more general FRW-like brane can be obtained using similar techniques as those presented here, but will be left for future work.

Before studying solutions including a curved brane, we briefly review the features of bulk solutions with curved slicing, which were studied in depth in [33].

Throughout this paper, a dot will denote a derivative with respect to the $u$ coordinate and a prime will denote a derivative with respect to the $\varphi$ coordinate, e.g.

$$
\dot{f}(u) \equiv \frac{d f(u)}{d u}, \quad F^{\prime}(\varphi) \equiv \frac{d F(\varphi)}{d \varphi} .
$$

With the restriction (2.15), the bulk Einstein's equations are:

$$
\begin{aligned}
2(d-1) \ddot{A}+\dot{\varphi}^{2}+\frac{2}{d} e^{-2 A} R^{(\zeta)} & =0, \\
d(d-1) \dot{A}^{2}-\frac{1}{2} \dot{\varphi}^{2}+V-e^{-2 A} R^{(\zeta)} & =0, \\
\ddot{\varphi}+d \dot{A} \dot{\varphi}-V^{\prime} & =0 .
\end{aligned}
$$

In [33], a formalism was developed by introducing three scalar functions of the bulk scalar field, $W(\varphi), S(\varphi)$ and $T(\varphi)$, which results in flow-like equations for the warp factor and scalar field,

$$
\begin{aligned}
\dot{A}(u) & =-\frac{1}{2(d-1)} W(\varphi(u)), \\
\dot{\varphi}(u) & =S(\varphi(u)), \\
R^{(\zeta)} e^{-2 A(u)} & =T(\varphi(u)) .
\end{aligned}
$$

The scalar functions $W(\varphi), S(\varphi), T(\varphi)$ may be defined piecewise in any region in which the scalar field $\varphi(u)$ is monotonic, and can eventually be glued together (imposing regularity) at extrema of the function $\varphi(u)$ (see $[33,50]$ for details).

We note that, for $R \neq 0$, our rewriting of the bulk Einstein's equation in terms of scalar functions differs from the first-order formalism obtained using Hamilton-Jacobi theory applied to holography [51]. In the flat case, the function $W(\varphi)$ is in one-to-one correspondence

\footnotetext{
${ }^{10}$ Here we have included the case $\kappa=0$ for completeness, but as we have explained in the previous subsection this can only lead to flat brane embeddings.
} 
with a solution of the radial Hamilton-Jacobi equation for Hamilton's principal function $\mathcal{S}(A, \varphi)$. For $R=0$ Hamilton-Jacobi's equation is separable and the solution can be written in the form $S=e^{d A} W(\varphi)$, where the superpotential $W$ satisfies equation (2.27). For $R \neq 0$ however, this is not the case: the Hamilton-Jacobi equation is non-separable, and a true first-order formalism can only be obtained starting from a non-trivial function $\mathcal{S}(A, \varphi)$. The reader is referred to [52] for a discussion of the first order Hamilton-Jacobi formalism for curved domain-walls in holography. Similar considerations apply to black-hole geometries [53]. A connection between our scalar functions and Hamilton-Jacobi formalism can be obtained proceeding along the lines of appendix B in [54].

Using the scalar functions $W, S, T$, we can write eqs. (2.18)-(2.20) as a system of first order differential equations in which $\varphi$ is the independent variable:

$$
\begin{aligned}
S^{2}-S W^{\prime}+\frac{2}{d} T & =0, \\
\frac{d}{2(d-1)} W^{2}-S^{2}-2 T+2 V & =0, \\
S S^{\prime}-\frac{d}{2(d-1)} S W-V^{\prime} & =0 .
\end{aligned}
$$

Flat space-time holographic RG flows are recovered by setting $T=0$ and $S=W^{\prime}$, resulting in the usual superpotential equation for $W(\varphi)$,

$$
\kappa=0: \quad \frac{d}{4(d-1)} W^{2}-\frac{1}{2} W^{\prime 2}=-V,
$$

Equation (2.25) is algebraic and it can be used to eliminate the function $T(\varphi)$ and we are left with the following two independent equations

$$
\begin{array}{r}
\frac{d}{2(d-1)} W^{2}+(d-1) S^{2}-d S W^{\prime}+2 V \\
=0, \\
S S^{\prime}-\frac{d}{2(d-1)} S W-V^{\prime}=0 .
\end{array}
$$

We will consider solutions in which we can identify a UV and and IR region, whose features we summarize below. Roughly, they can be identified with the regions where the scale factor $e^{A(u)}$ becomes large (UV) or small (IR).

UV region. This is the region where the scale factor becomes large and the geometry asymptotes to the conformal boundary of Anti-de Sitter space. Generically, this is realised when $\varphi(u)$ approaches a maximum of the scalar potential (which we set at $\varphi=0$ for simplicity). Around the maximum, the potential has an expansion of the form

$$
V(\varphi)=-\frac{d(d-1)}{\ell^{2}}+\frac{m^{2}}{2} \varphi^{2}+\mathcal{O}\left(\varphi^{3}\right)
$$

where $m^{2}<0$. The boundary is approached as $u \rightarrow-\infty$, and the solution takes the asymptotic form

$$
A(u)=-\frac{u}{\ell}+\ldots, \quad \varphi(u)=\varphi_{-} \ell^{\Delta_{-}} e^{\Delta_{-} u / \ell}+\ldots \quad u \rightarrow-\infty,
$$


where $\Delta_{-}=d / 2-\sqrt{d^{2} / 4+m^{2} \ell^{2}}$ and $\varphi_{-}$is an integration constant. In the AdS/CFT dictionary, the constant $\varphi_{-}$is interpreted, in the dual field theory, as the value of the source for a gauge-invariant operator $\mathcal{O}$ whose dimension is $\Delta_{+} \equiv d-\Delta_{-}$. This geometry is dual to an RG flow away from a UV conformal fixed point, driven by a deformation by the relevant operator $\mathcal{O}$. As $u$ increases, $\varphi$ grows, backreaction starts becoming important, and the geometry deviates more and more from AdS.

A detailed description of the solution in the UV, both in terms of the functions $W(\varphi)$, $S(\varphi)$ and $T(\varphi)$ and of the scale factor $A(u)$ and scalar field profile $\varphi(u)$, can be found in appendix B. Here we point out a few important features:

- To leading order as $\varphi \rightarrow 0$, the behaviour of $W$ and $S$ is universal and it is the same as in the zero-curvature case, ${ }^{11}$

$$
W=\frac{2(d-1)}{\ell}+\frac{\Delta_{-}}{2 \ell} \varphi^{2}+\ldots, \quad S \simeq W^{\prime}=\Delta_{-} \varphi+\ldots, \quad \varphi \rightarrow 0 .
$$

The precise behavior including subleading terms can be found in appendix B.

- The two first order differential equations (2.28)-(2.29) contain two independent integration constants $C$ and $\mathcal{R}$. They appear as coefficients of subleading (with respect to those in (2.32)) terms $W_{\text {sub }}(\varphi), S_{\mathrm{sub}}(\varphi)$ in the expansion of $W$ and $S$ around the $\mathrm{UV} \varphi=0$ :

$$
W_{\text {sub }}=\frac{\mathcal{R}}{d \ell}|\varphi|^{\frac{2}{\Delta_{-}}}+\ldots+\frac{C}{\ell}|\varphi|^{\frac{d}{\Delta_{-}}}+\ldots, \quad \varphi \rightarrow 0 .
$$

and similarly for $S_{\text {sub }}$.

Together with $\varphi_{-}$, the parameters $\mathcal{R}$ and $C$ exhaust the three integration constants of the third order system of Einstein equations (2.18)-(2.19). The constants $C$ and $\mathcal{R}$ fix the "dimensionless" (i.e. in units of $\varphi_{-}$) boundary curvature and vacuum expectation value of the dual operator,

$$
\langle\mathcal{O}\rangle_{-}=\frac{C d}{\Delta_{-}}\left|\varphi_{-}\right|^{\Delta_{+} / \Delta_{-}}, \quad \mathcal{R}=R^{(\zeta)}\left|\varphi_{-}\right|^{-2 / \Delta_{-}}
$$

As we have discussed, the properties of the UV part of the solution are universal, and depend on the curvature only at subleading orders. In contrast, properties of the solution in the region, where the scale factor becomes small (corresponding to the IR of the dual QFT) are very different depending on the sign of the curvature. Below we review the structure of the regular IR region for zero, positive and negative curvature. More details can be found in [50] and [33].

\footnotetext{
${ }^{11}$ Here we only give the behavior of the " $W_{-}$" type function, which in AdS/CFT language (and with the standard quantisation) corresponds to a deformation of the CFT by a source. For completeness, the reader will also find in appendix B the " $W_{+}$" type function, which corresponds to a deformation by a vev with no source term.
} 


\section{IR region, $\kappa=0$.}

\section{Regular AdS interior}

We start by recalling the situation for zero curvature. In the flat case, a regular interior can arise only as a new asymptotically AdS region, where $e^{A(u)} \sim e^{-2 u / \ell_{\mathrm{IR}}}$ vanishes as $u \rightarrow+\infty$. This can occur as $\varphi$ approaches a minimum $\varphi_{\mathrm{IR}}$ of the bulk potential, around which

$$
V(\varphi) \simeq-\frac{d(d-1)}{\ell_{\mathrm{IR}}^{2}}+\frac{m_{\mathrm{IR}}^{2}}{2}\left(\varphi-\varphi_{\mathrm{IR}}\right)^{2}+\ldots
$$

with $m_{\mathrm{IR}}^{2}>0$. Unlike the case for a UV maximum, where all $W$ solutions have the same behaviour (2.32), here only a single solution can reach a minimum. The regular solution reaching the minimum behaves close to $\varphi_{\mathrm{IR}}$ as

$$
W_{\mathrm{IR}}(\varphi) \simeq \frac{2(d-1)}{\ell_{\mathrm{IR}}}+\frac{\Delta_{\mathrm{IR}}^{-}}{2 \ell_{\mathrm{IR}}}\left(\varphi-\varphi_{\mathrm{IR}}\right)^{2}+\ldots
$$

where $\Delta_{\mathrm{IR}}^{-}=d / 2-\sqrt{d^{2} / 4+m^{2} \ell^{2}}<0$. We can see that when $V$ has a minimum, $W$ has a maximum. The corresponding geometry approaches the Poincaré horizon of AdS as $u \rightarrow+\infty$,

$$
A(u)=-\frac{u}{\ell_{\mathrm{IR}}}+\ldots, \quad \varphi=\varphi_{\mathrm{IR}}-\bar{\varphi} \exp \left[\frac{\Delta_{\mathrm{IR}}^{-} u}{\ell_{\mathrm{IR}}}\right], \quad u \rightarrow+\infty .
$$

where $\bar{\phi}$ is a constant, interpreted as the (irrelevant) coupling in the IR CFT.

2. $\varphi \rightarrow \infty$ : good singularities

If $\varphi$ does not reach a fixed point, it will flow all the way to $\varphi \rightarrow \pm \infty$. In this case, the solution has a naked singularity in the IR. Nevertheless, this can be an acceptable IR region if it satisfies certain requirements, which can be translated into the asymptotic behaviour of the function $W$ as $\varphi \rightarrow \infty$. Below we summarize these requirements for a potential whose asymptotic behaviour for large $\varphi$ is parametrized by a positive exponential (we discuss the case $\varphi \rightarrow+\infty$ for concreteness),

$$
V \simeq-V_{\infty} e^{b \varphi} \quad \varphi \rightarrow+\infty
$$

For such a potential, the asymptotic solutions $W(\varphi)$ as $\varphi \rightarrow \infty$ fall in two classes:

- Generic

$$
W(\varphi) \simeq W_{0} e^{Q \varphi}, \quad Q \equiv \sqrt{\frac{d}{2(d-1)}},
$$

where $W_{0}$ is an arbitrary constant. Notice that the exponential behavior does not depend on the parameter $b$ in the potential in equation (2.38). Indeed the potential $V$ affects this class of solutions at subleading orders. 


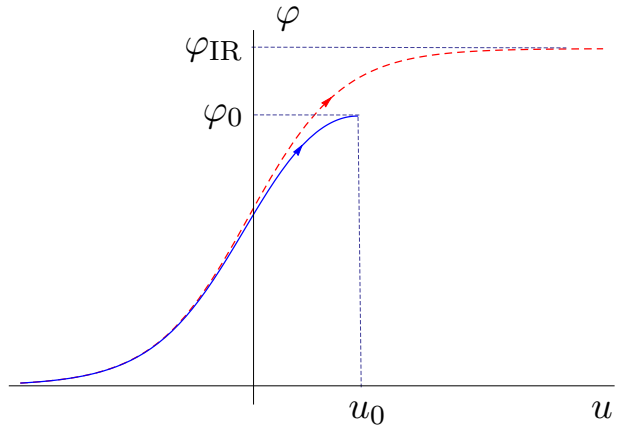

(a)

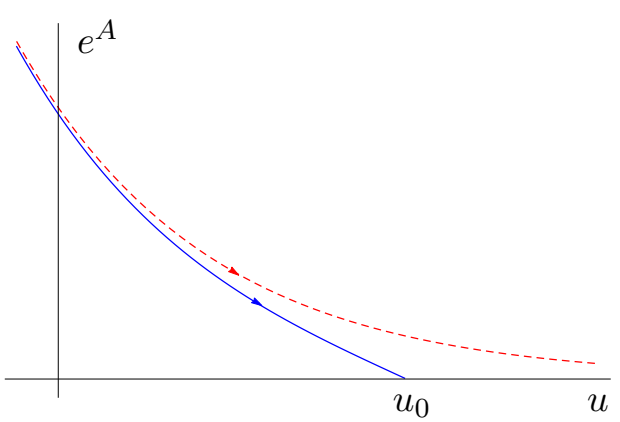

(b)

Figure 2. The scalar field (a) and scale factor (b) profiles of a positive curvature RG flow geometry (solid lines). Both the scale factor and the scalar field have an IR end point at $u=u_{0}, \varphi=\varphi_{0}$. The corresponding zero-curvature solutions extending to the conformal fixed point $\varphi=\varphi_{\mathrm{IR}}$ (dashed lines) are shown for comparison.

- Special

$$
W(\varphi) \simeq \sqrt{\frac{8 V_{\infty}}{4 Q^{2}-b^{2}}} e^{\frac{b}{2} \varphi} .
$$

This solution exists only for $b<2 Q$, is isolated, as it has no tunable integration constants, and has a softer exponential growth than all of the solutions of type (2.39).

One can argue that only the isolated special solution has a meaningful holographic interpretation. For example, one can impose Gubser's criterion [25], which requires that one can cloak the singularity by an arbitrarily small horizon. This is true for the solution (2.40), but not for the solutions of type (2.39). Notice that the existence of the special solution puts an upper bound $b<2 Q$ on the asymptotic growth rate of the bulk potential.

IR region, $\boldsymbol{\kappa}>\mathbf{0}$. For $R^{(\zeta)}>0$, it was shown in [33] that regular geometries reach a point $u=u_{0}$, at which $\dot{\varphi}=0$ and $e^{2 A}$ vanishes as $\left(u_{0}-u\right)^{2}$. This is a regular endpoint in the Euclidean case, and a horizon in the Lorentzian signature. This point corresponds to the extreme infrared, since the whole space-time shrinks to zero size. Figure 2 shows a comparison between a positive curvature flow and the corresponding regular zero-curvature solution, in a case where the latter arrives at an IR fixed point at finite $\varphi$.

A few properties of these solutions are listed below:

- The dimensionless parameters of these solutions are fixed by specifying the value of the scalar field $\varphi_{0}$ at the IR endpoint, and imposing regularity. This determines the dimensionless UV parameters $\mathcal{R}$ and $C$.

- Fixing $\varphi_{0}$ leaves a one-parameter family of solutions parametrized by $\varphi_{-}$. The latter can be taken as setting the scale for the all dimensionful features of the solution 


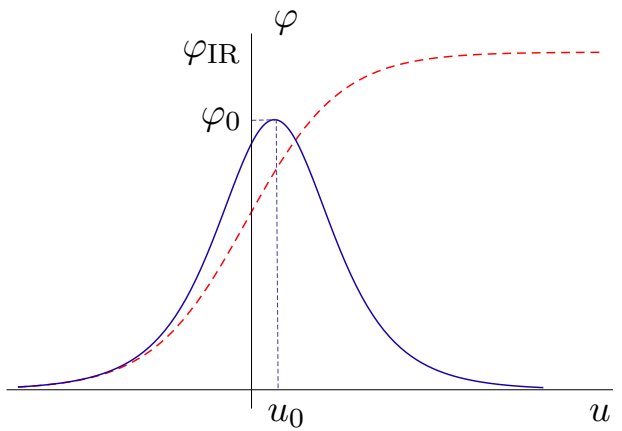

(a)

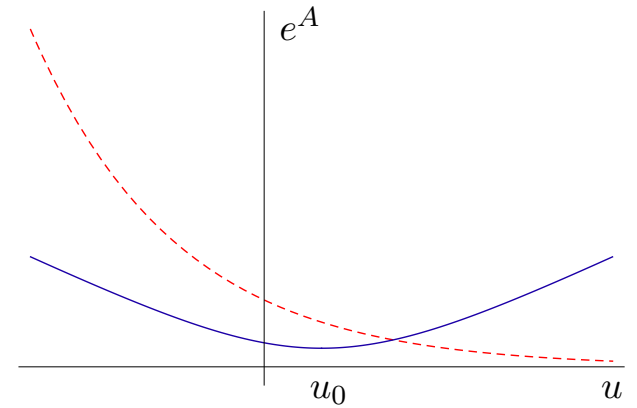

(b)

Figure 3. The scalar field (a) and scale factor (b) profiles of a negative curvature RG flow geometry (solid lines). Both the scale factor and the scalar field have a turning point at the bottom of the throat, $u=u_{0}$. The corresponding zero-curvature solutions extending to the conformal fixed point $\varphi=\varphi_{\mathrm{IR}}$ (dashed lines) are shown for comparison. The solution is symmetric around $u_{0}$.

(curvature, operator vev). Therefore, fixing $\varphi_{-}$and varying the endpoint in field space $\varphi_{0}$, one can scan over the range of positive curvature solutions.

- For strictly positive $R^{(\zeta)}>0$, the endpoint $\varphi_{0}$ cannot coincide with an extremum of the potential: this would lead to either infinite curvature (maximum) or zero curvature (minimum).

- At the IR endpoint, $W(\varphi)$ diverges as $\left|\varphi_{0}-\varphi\right|^{-1 / 2}$ and $S(\varphi)$ vanishes as $\left|\varphi_{0}-\varphi\right|^{1 / 2}$.

IR region, $\boldsymbol{\kappa}<\mathbf{0}$. In this case, the scale factor never shrinks to zero. Instead, the geometry reaches a throat of finite size where both $\dot{\varphi}=0$ and $\dot{A}=0$, and the scale factor takes a minimum value $A\left(u_{0}\right) \neq 0$. The two sides of the throat connect to different regions of the asymptotic AdS boundary. As explained in [31] in the case of constant potential, and noted also in [33] in the general case, this corresponds to the fact that the UV theory has a codimension-one defect. These features of negatively curved flows can be seen in figure 3:

- As in the $\kappa>0$ case, the value of the scalar field $\varphi_{0}$ at the bottom of the throat fixes all integration constants in the solution (except $\varphi_{-}$which can always be chosen independently).

- Also in this case, the turning point $\varphi_{0}$ cannot coincide with an extremum of the potential.

- At the IR endpoint, both $W(\varphi)$ and $S(\varphi)$ vanish as $\left|\varphi_{0}-\varphi\right|^{1 / 2}$.

The properties of the solutions we have described in this subsection are summarized in figure 4, where we sketch the behavior of different solutions for the scalar function $W(\varphi)$ in a model in which the potential $V(\varphi)$ has a maximum at $\varphi=0$ and a minimum at $\varphi=1$. As one can see, all solutions connect to the UV fixed point at $\varphi=0$. The flat 


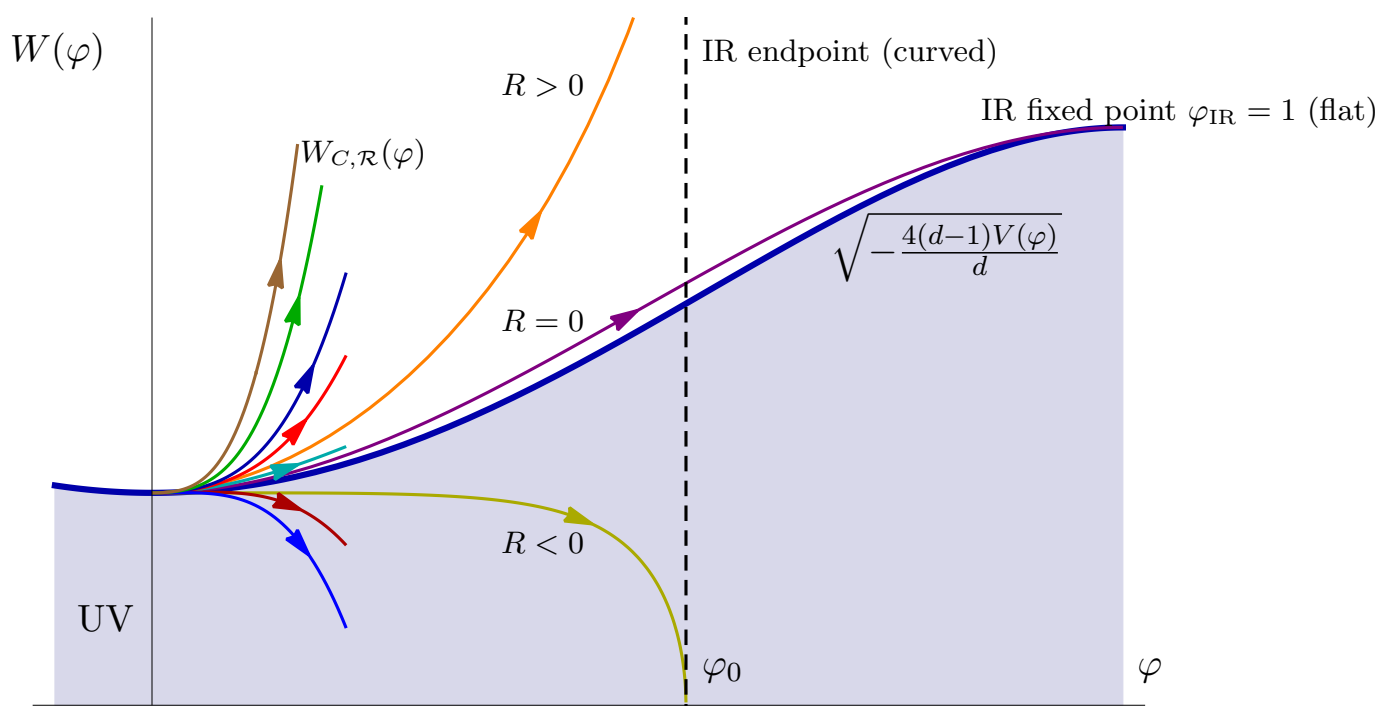

Figure 4. Sketch of different solutions for $W(\varphi)$, for various curvatures, in a theory with a UV and an IR fixed point at $\varphi=0$ and $\varphi=1$, respectively. Of all the solution starting from the UV fixed point, three are followed to the IR endpoint, which can be at the conformal fixed point $(R=0)$ or an intermediate point $0<\varphi_{0}<1$. For $R^{(\zeta)} \geq 0$ the shaded region below the blue curve cannot be accessed.

solution reaches the IR conformal fixed at $\varphi_{\mathrm{IR}}=1$; the regular curved $\mathrm{RG}$ flow solutions reach an endpoint or a throat at $0<\varphi_{0}<1$. An important role is played by the curve $B(\varphi) \equiv \sqrt{-4(d-1) V(\varphi) / d}$, displayed in dark blue in the figure: for zero and positive curvature, this curve mark the boundary of a forbidden region (shaded area in the figure) which $W(\varphi)$ cannot reach. On the other hand, for $R<0$ the solution can reach into the forbidden region.

We end this review section with the remark that solutions may exhibit one or several bounces, i.e. regular points where $\dot{\varphi}=0$ but $\dot{A} \neq 0[50,55]$. These can occur in principle at any point where $V^{\prime} \neq 0$, and they where found to be a generic feature of curved RG-flow solutions [33]. The possibility of bounces has to be taken into account when we introduce the brane.

The details of the behavior of the functions $W, S$ and $T$ near an IR endpoint or a bounce are given in appendix C.

\subsection{The junction conditions}

We are now in the position to introduce the brane as an interface between two geometries of the form (2.4). The dynamics of the brane is encoded in the junction conditions, as we explain below.

We consider solutions where the geometry to one side of the brane connects to an UV-type region, and the other to an IR-type region. To distinguish between the two sides, we label the metric and scalar field on the two sides of the brane by $g_{a b}^{\mathrm{UV}}, g_{a b}^{\mathrm{IR}}$ and $\varphi^{\mathrm{UV}}, \varphi^{\mathrm{IR}}$. 
When a quantity $X$ exhibits a jump across the position of the brane, this will be written as $[X]_{\mathrm{IR}}^{\mathrm{UV}}$. The Israel matching conditions then result in the following two requirements:

1. The metric and scalar field are continuous across the brane:

$$
\left[g_{a b}\right]_{\mathrm{IR}}^{\mathrm{UV}}=0, \quad[\varphi]_{\mathrm{UV}}^{\mathrm{IR}}=0
$$

2. The extrinsic curvature as well as the normal derivative of $\varphi$ are discontinuous:

$$
\left[K_{\mu \nu}-\gamma_{\mu \nu} K\right]_{\mathrm{UV}}^{\mathrm{IR}}=\frac{1}{\sqrt{-\gamma}} \frac{\delta S_{\text {brane }}}{\delta \gamma^{\mu \nu}}, \quad\left[n^{a} \partial_{a} \varphi\right]_{\mathrm{UV}}^{\mathrm{IR}}=-\frac{1}{\sqrt{-\gamma}} \frac{\delta S_{\text {brane }}}{\delta \varphi} .
$$

Here $\gamma_{\mu \nu}=e^{2 A(u)} \zeta_{\mu \nu}$ is the induced metric, $K_{\mu \nu}$ is the extrinsic curvature of the brane with $K=\gamma^{\mu \nu} K_{\mu \nu}$ the trace, and $n^{a}$ is a unit vector normal to the brane with orientation towards the IR.

For our setup given in (2.3) the equations (2.42) become

$$
\begin{aligned}
{\left[K_{\mu \nu}-\gamma_{\mu \nu} K\right]_{\mathrm{UV}}^{\mathrm{IR}}=} & {\left[\frac{1}{2} W_{B}(\varphi) \gamma_{\mu \nu}+U(\varphi) G_{\mu \nu}^{B}-\frac{1}{2} Z(\varphi)\left(\partial_{\mu} \varphi \partial_{\nu} \varphi-\frac{1}{2} \gamma_{\mu \nu}(\partial \varphi)^{2}\right)\right.} \\
& \left.+\left(\gamma_{\mu \nu} \gamma^{\rho \sigma} \nabla_{\rho} \nabla_{\sigma}-\nabla_{\mu} \nabla_{\nu}\right) U(\varphi)\right]_{\varphi_{\star}} \\
{\left[n^{a} \partial_{a} \varphi\right]_{\mathrm{UV}}^{\mathrm{IR}}=} & {\left[\frac{d W_{B}}{d \varphi}-\frac{d U}{d \varphi} R_{B}+\frac{1}{2} \frac{d Z}{d \varphi}(\partial \varphi)^{2}-\frac{1}{\sqrt{-\gamma}} \partial_{\mu}\left(Z(\varphi) \sqrt{-\gamma} \gamma^{\mu \nu} \partial_{\nu} \varphi\right)\right]_{\varphi_{\star}}, }
\end{aligned}
$$

where $\nabla_{\mu}, G_{\mu \nu}^{B}$ and $R_{B}$ are the covariant derivative, Einstein tensor and Ricci scalar computed from the induced metric and $\varphi_{\star}\left(x^{\mu}\right) \equiv \varphi\left(u_{\star}, x^{\mu}\right)$ is the scalar field at the position of the brane. Furthermore, for our setting we find:

$$
K_{\mu \nu}=\dot{A} \gamma_{\mu \nu}, \quad K_{\mu \nu}-\gamma_{\mu \nu} K=-(d-1) \dot{A} \gamma_{\mu \nu}=\frac{1}{2} W \gamma_{\mu \nu}, \quad n^{a} \partial_{a} \varphi=\dot{\varphi}=S .
$$

Further, recall that $R_{\mu \nu}^{B}=R_{\mu \nu}^{(\zeta)}$ and $R_{B}=e^{-2 A} R^{(\zeta)}$, hence

$$
G_{\mu \nu}^{B}=G_{\mu \nu}^{\zeta}=\frac{1}{2}(2-d) \kappa e^{-2 A} \gamma_{\mu \nu}=\frac{2-d}{2 d} T \gamma_{\mu \nu}
$$

where we have used the definitions (2.21)-(2.23). We introduce $W_{\mathrm{UV}}, S_{\mathrm{UV}}$ and $W_{\mathrm{IR}}, S_{\mathrm{IR}}$ as the functions $W$ and $S$ for the UV and the IR regions, respectively. Using these quantities we can then write the junction conditions (2.43)-(2.44) as:

$$
\begin{aligned}
W_{\mathrm{IR}}-\left.W_{\mathrm{UV}}\right|_{\varphi_{*}} & =W_{B}+\left.\frac{(2-d)}{d} U T\right|_{\varphi_{\star}}, \\
S_{\mathrm{IR}}-\left.S_{\mathrm{UV}}\right|_{\varphi_{*}} & =W_{B}{ }^{\prime}-\left.U^{\prime} T\right|_{\varphi_{\star}} .
\end{aligned}
$$

From the continuity of the metric (2.41), we can infer that the scale factor is continuous across the brane and the same is true for the function $T(\varphi)$,

$$
T_{\mathrm{UV}}\left(\varphi_{\star}\right)=T_{\mathrm{IR}}\left(\varphi_{\star}\right)
$$


Using the continuity of $T$ and $\varphi$ across the brane, it follows from (2.25) that

$$
\frac{d}{2(d-1)} W_{\mathrm{UV}}^{2}-\left.S_{\mathrm{UV}}^{2}\right|_{\varphi_{\star}}=\frac{d}{2(d-1)} W_{\mathrm{IR}}^{2}-\left.S_{\mathrm{IR}}^{2}\right|_{\varphi_{\star}}
$$

We can write the conditions $(2.47)-(2.48)$ as

$$
\begin{aligned}
\left.W_{\mathrm{UV}}\right|_{\varphi_{*}} & =W_{\mathrm{IR}}-W_{B}-\left.\frac{2-d}{d} U T_{\mathrm{IR}}\right|_{\varphi_{\star}} \\
\left.S_{\mathrm{UV}}\right|_{\varphi_{*}} & =S_{\mathrm{IR}}-W_{B}^{\prime}+\left.U^{\prime} T_{\mathrm{IR}}\right|_{\varphi_{\star}} .
\end{aligned}
$$

From the equation of motion we can write

$$
Q^{2} W_{\mathrm{UV}}^{2}-S_{\mathrm{UV}}^{2}-2 T_{\mathrm{UV}}+2 V=0
$$

where $Q^{2}=\frac{d}{2(d-1)}$. Using eqs. (2.51)-(2.52) and using the fact that $T$ and $\varphi$ are continuous, we can express everything in terms of IR quantities. Using also $Q^{2} W_{\mathrm{IR}}^{2}-S_{\mathrm{IR}}^{2}-2 T_{\mathrm{IR}}+2 V=0$ and after a bit of algebra we obtain the condition:

$$
\begin{aligned}
{\left[-2 Q^{2} W_{\mathrm{IR}}\left(W_{B}\right.\right.} & \left.+\frac{2-d}{d} U T_{\mathrm{IR}}\right)+Q^{2}\left(W_{B}+\frac{2-d}{d} U T_{\mathrm{IR}}\right)^{2} \\
+ & \left.+2 S_{\mathrm{IR}}\left(W_{B}^{\prime}-U^{\prime} T_{\mathrm{IR}}\right)-\left(W_{B}^{\prime}-U^{\prime} T_{\mathrm{IR}}\right)^{2}\right]_{\varphi_{\star}}=0 .
\end{aligned}
$$

Notice that all functions of $\varphi$ involved in this equation are in principle known, in terms of a few input quantities: $V, W_{B}$ and $U$ are fixed by the choice of the action; $W_{\mathrm{IR}}, S_{\mathrm{IR}}$ and $T_{\mathrm{IR}}$ are determined by regularity, plus the choice of the endpoint $\varphi_{0}$ of the IR solution. Therefore, once the underlying model and $\varphi_{0}$ are chosen, (2.54) provides a transcendental equation for the brane position $\varphi_{\star}$, which generically has a finite number of solutions (including the possibility of no solution).

Once $\varphi_{\star}$ is determined, we can use equations $(2.51)-(2.52)$ as initial conditions for $W_{\mathrm{UV}}$ and $S_{\mathrm{UV}}$, to be used in the system of differential equations (2.28)-(2.29) which determines the solution for $W$ and $S$ in the UV and the corresponding values of $\mathcal{R}$ and $C$ (the dimensionless curvature and vev parameters).

To summarize, one can use the following algorithmic procedure to solve the system from the IR, across the brane, to the UV:

$$
\text { choice of } \varphi_{0} \rightarrow W_{\mathrm{IR}}, S_{\mathrm{IR}} \rightarrow \varphi_{\star} \rightarrow W_{\mathrm{UV}}, S_{\mathrm{UV}} \rightarrow \mathcal{R}, C
$$

The only control parameter here is $\varphi_{0}$, which determines everything else. In particular, the choice of $\varphi_{0}$ at an IR extremum of the potential would result in the flat-sliced solution with $\mathcal{R}=0$. For the case when the flat solution IR is reached as $\varphi \rightarrow \infty$, things are more subtle, as we will see in section 4 . 


\subsection{Junction rules}

Here we discuss what are the geometric rules to patch together two geometries across the brane, and which types of junctions give rise to a sensible holographic interpretation.

In the positive or zero curvature case, the flow of $A(u)$ is monotonically decreasing from the UV to the IR. Since $\dot{A} \propto-W$, the scalar function $W(\varphi)$ cannot change sign. ${ }^{12}$ At the junction, we must require that one side of the brane actually connects to a UV region, and the other to an IR region. This implies that the flow of $A(u)$ must not change direction, i.e. $\dot{A}$ should not change sign, across the brane. Since at the brane position $\dot{A}_{\mathrm{UV}} \propto-W_{\mathrm{UV}}\left(\varphi_{\star}\right)$ and $\dot{A}_{\mathrm{IR}} \propto-W_{\mathrm{IR}}\left(\varphi_{\star}\right)$, we must discard solutions in which $W_{\mathrm{UV}}\left(\varphi_{\star}\right)$ and $W_{\mathrm{IR}}\left(\varphi_{\star}\right)$ have opposite signs. If that were the case, we would be joining two UV or two IR regions.

The above constraint does not apply to solutions with negatively curved slices: in this case $\dot{A}$ (and $W$ ) can change sign in the bulk, and there is no reason why it should not change sign across the brane. In fact, in this case, both sides of the brane eventually reach a UV region.

Next, since we will solve the matching conditions in field space, rather than in coordinate space, we need to understand towards which side (i.e. direction of increasing or decreasing $\varphi$ away from $\varphi_{\star}$ ) one should follow the solution $W_{\mathrm{UV}}(\varphi)$ on the UV side of the brane. As we discussed at the end of the previous subsection, if we start from the IR side of the solution, the junction conditions determine the pair of initial conditions $\left(S_{\mathrm{UV}}\left(\varphi_{\star}\right), W_{\mathrm{UV}}\left(\varphi_{\star}\right)\right)$ for the system $(2.51)-(2.52)$, and we need to know if we should keep the solution for $\varphi>\varphi_{\star}$ or $\varphi<\varphi_{\star}$. To understand what the correct choice is, recall that in our conventions the coordinate $u$ runs in the same direction on both sides of the brane, and we take it to be increasing from the UV to the IR. therefore if the brane is at $u_{\star}$, the IR side is $u>u_{\star}$, and the UV side is $u<u_{\star}$. Then, to be consistent with this choice, it is the sign of $\dot{\varphi}\left(u_{\star}\right) \equiv S_{\mathrm{UV}}\left(\varphi_{\star}\right)$ which decides which one is the right direction to follow on the UV side:

- If $S_{\mathrm{UV}}\left(\varphi_{\star}\right)>0$, then $\dot{\varphi}\left(u_{\star}\right)>0$ at the brane, and we should take the UV solution such that $\varphi$ increases towards the brane, i.e. the solution $W_{\mathrm{UV}}, S_{\mathrm{UV}}$ for $\varphi_{\mathrm{UV}}<\varphi_{\star}$.

- Conversely, if $S_{\mathrm{UV}}\left(\varphi_{\star}\right)<0$, we should take the other part of the solution, the one with $\varphi_{\mathrm{UV}}>\varphi_{\star}$.

This junction rule is summarized graphically in figure 5 .

Finally, due to stability requirements of the solution, some care is needed when choosing the combination of bulk and brane potentials appearing in the action. Because we will not pursue phenomenological applications here, and in general we will not worry about whether the functions chosen can lead to physics compatible with observation (e.g. the presence of four-dimensional gravity in the brane), we will try to require that at least the flat solutions be free of ghosts and tachyonic instabilities. Although this does not straightforwardly

\footnotetext{
${ }^{12}$ Since the overall sign of $W$ can be changed by sending $u \rightarrow-u$, we will always choose $W>0$ for definiteness.
} 

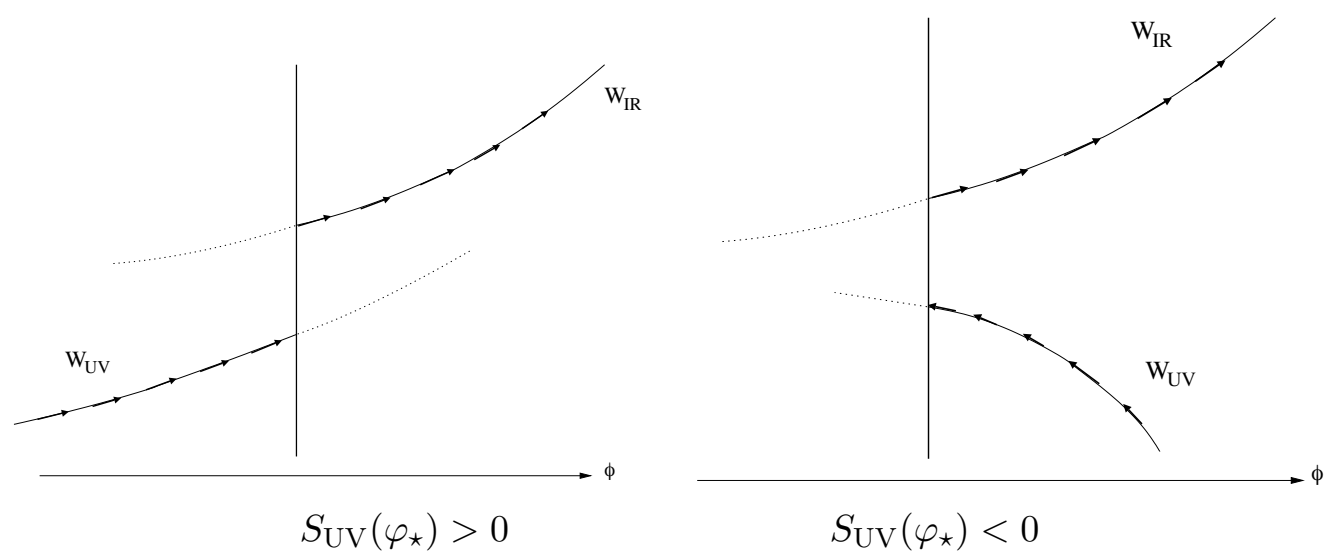

Figure 5. Junction rules in field space. The vertical solid line indicates the locus of the brane.

guarantee stability of the curved solutions, there are strong indications that this is the case, at least for the positive curvature solutions, as discussed in section 4.5 of [33].

That there are no instabilities in the bulk is automatically guaranteed if the scalar field kinetic term has the correct sign, and there are no violations of the BF bound in the UV or in the IR. However, some unstable modes can still arise due to the brane fluctuations. For the flat case, the analysis of [20] showed that there are very simple sufficient conditions which guarantee the absence of ghosts and tachyonic instabilities for the self-tuning flat brane solutions. These are:

$$
\begin{aligned}
U\left(\varphi_{\star}\right)>0, \quad Z\left(\varphi_{\star}\right) & >0 \\
\frac{W_{B}\left(\varphi_{\star}\right)}{W_{\mathrm{IR}}\left(\varphi_{\star}\right) W_{\mathrm{UV}}\left(\varphi_{\star}\right)} & >\frac{U\left(\varphi_{\star}\right)}{3}, \\
Z\left(\varphi_{\star}\right)\left(\frac{W_{B}\left(\varphi_{\star}\right)}{W_{\mathrm{IR}}\left(\varphi_{\star}\right) W_{\mathrm{UV}}\left(\varphi_{\star}\right)}-\frac{U\left(\varphi_{\star}\right)}{3}\right) & >\left(\frac{d U}{d \varphi}\right)_{\varphi_{\star}}^{2}, \\
W_{\mathrm{IR}}^{\prime \prime}\left(\varphi_{\star}\right)-W_{\mathrm{UV}}^{\prime \prime}\left(\varphi_{\star}\right) & <W_{B}^{\prime \prime}\left(\varphi_{\star}\right) .
\end{aligned}
$$

Conditions (2.56) are also necessary: violating either of them would guarantee the presence of either a spin- 2 or a spin-0 ghost. On the other hand, the other conditions are only sufficient, but if they are violated the stability analysis becomes much more involved, and requires a detailed perturbation analysis of the full bulk solution.

Since here we will not aim to build explicit, realistic phenomenological models, we will not always strictly enforce the conditions (2.57)-(2.59), not to limit too much the scope of the examples we study.

\subsection{Curved CFT boundary metrics vs. variable scalar sources}

In this section we briefly comment on a possible alternative realisation of the solutions described so far, in terms of a dual CFT living in Minkowski space, but coupled to a non-trivial time-dependent (for dS branes) or space-dependent (for AdS) external source. 
In the UV region, the bulk metric (2.4) takes the asymptotic form (2.5), where $\zeta_{\mu \nu}^{\mathrm{UV}}$ is the metric to which the dual CFT is coupled, and in the examples described above is (A) $\mathrm{dS}_{d}$. It is well known (see e.g. [35]) that one can foliate $\mathrm{AdS}_{d+1}$ by either Minkowski, $\mathrm{dS}_{d}$ or $\mathrm{AdS}_{d}$. From the bulk point of view, these choices only differ by a coordinate transformation. From the dual field theory point of view, however, different coordinate choices lead to different physical theories, as the appropriate coordinate transformation acts non-trivially on the conformal boundary and it changes both the metric and the scalar sources. ${ }^{13}$ Therefore, we can use these coordinate transformations to find new solutions with a different holographic interpretation. In the rest of this section we consider the case of a dS brane for definiteness. The case of AdS-slicing is more subtle because the slices are non-compact and one can reach the boundary along each radial slice. This means that we need boundary conditions also at the boundary of the slices. This is related to the fact that, as we explained in section 2.2, when discussing the case $\kappa<0$, there are two (disconnected) boundary regions, which correspond to the field theory dual theory having a defect. These issues are discussed in [33] and are similar to those arising in the case of the Janus solution, [31].

Let us therefore consider dS-sliced domain walls. Going from a curved to a flat foliation by a coordinate transformation is only possible if the bulk is exactly $\mathrm{AdS}_{d+1}$, not only asymptotically. However, we can still perform a coordinate transformation to new radial and time coordinates $(\rho, t)$ of the form

$$
u=f(\rho, t), \quad \tau=g(\rho, t),
$$

such that close to the UV boundary it changes a dS slicing into a flat one. If we take $\tau$ to be the conformal time coordinate in the de Sitter metric ansatz, i.e. $\zeta_{\mu \nu}^{\mathrm{UV}}=\frac{H^{2}}{\tau^{2}} \eta_{\mu \nu}, f$ and $g$ can be two arbitrary smooth functions constrained only by demanding that their asymptotic form leads to a flat UV metric in the new coordinates $(\rho, t), \zeta_{\mu \nu}^{\mathrm{UV}}=\eta_{\mu \nu}$, in the limit $u \rightarrow-\infty$. This imposes the following constraints,

$$
f(\rho, t) \rightarrow \rho-\ell \ln \left(-\frac{t}{\ell}\right), \quad g(\rho, t) \rightarrow t, \quad \rho \rightarrow-\infty,
$$

where $-\infty<t<0$ (see e.g. section 4.4 of [33] for the full coordinate transformation, from which the limits (2.61) can be easily obtained).

In the original coordinates $(u, \tau)$ the brane was located at the equilibrium position $u=u_{\star}$. In the new coordinates this will result in a non-trivial trajectory,

$$
f(\rho, t)=u_{\star} \quad \Rightarrow \quad \rho=\rho_{\star}(t) .
$$

The important point is that, by construction,

1. The junction conditions are still satisfied, since they have tensorial nature;

\footnotetext{
${ }^{13} \mathrm{~A}$ well-known example of this phenomenon is the difference between global and Poincaré AdS coordinates, which give a different structure of the conformal boundary metric and describe a dual field theory on $R \times S^{d-1}$, and $R^{1, d-1}$, respectively.
} 
2. The induced metric on the brane is diffeomorphic to the original one before the change of coordinates.

This implies that we have an alternative embedding of the same dS brane, which is now moving in an asymptotically AdS space-time whose asymptotic boundary has a flat metric source. The dual field theory lives therefore in flat space.

This is not the end of the story however: recall that the bulk has also a non-trivial scalar field profile. In the old coordinates $(u, \tau)$ this is described by a function $\varphi(u)$, which becomes a time-dependent function $\varphi(\rho, t)$ in the new coordinates. This has an important implication: writing the near-boundary scalar field asymptotics (2.31) in the new coordinates using (2.61), we find

$$
\varphi(\rho, t) \simeq \varphi_{-}\left(\frac{\ell}{|t|}\right)^{\Delta_{-}} \ell^{\Delta_{-}} e^{\Delta_{-} \rho / \ell}+\ldots \quad \rho \rightarrow-\infty .
$$

In the holographic dictionary, this implies that the CFT is coupled to a time-dependent external source

$$
j=\left(\frac{\ell}{|t|}\right)^{\Delta_{-}} \varphi_{-}
$$

The source is switched on from $j=0$ at early times and increases in time as a powerlaw. Thus, in this language, cosmological de Sitter expansion of the brane is driven by a time-dependent source in a flat-space CFT. This gives an alternative (and, from the CFT standpoint, inequivalent) description of the solutions we are discussing.

Notice that the only case in which one can embed a dS brane in a flat CFT with no sources is the Karch-Randall-like setup, where the bulk is AdS (in any coordinates), with no scalar field. In this case a non-zero brane tension and/or induced Einstein term generically require patching together two AdS spaces with different curvatures, as was the case in $[36,37,40]$.

\section{Solutions with IR fixed points}

We now present implementations of the self-stabilisation mechanism in several example models. Thus, here and in what follows we set $d=4$. A particular model will be characterised by a choice of bulk potential $V(\varphi)$ and the brane quantities $W_{B}(\varphi)$ and $U(\varphi)$. While the functions $V, W_{B}$ and $U$ should be determined from a microscopic model, this goes beyond the scope of this paper. Instead, the functions will be chosen by hand and the consequences for self-stabilisation studied. Also, we will not be interested in constructing phenomenologically viable models, as this also goes beyond the scope of this investigation. The main goal of this section is to study the viability and efficacy of self-stabilisation in this holographic setting with non-zero UV curvature. In particular, we wish to answer the following questions:

1. How do self-stabilising solutions with non-zero UV curvature differ from the selftuning solutions with vanishing UV curvature studied in [20]? 
2. How does the brane curvature $R_{B}$ depend on the UV curvature $R^{(\zeta)}$ ? E.g. can $R_{B}$ be small while $R^{(\zeta)}$ is large (in suitable units) and vice versa.

3. Can the brane curvature $R_{B}$ be small in units of the 4 d Planck mass $M_{4}$ on the brane without the need of tuning of model parameters?

Finally, since analytical solutions are out of the question for generic setups, the results presented in this section come from numerical studies.

In the first part of the numerical investigation, we choose a generic bulk potential and focus on a region containing a maximum and bounded by its two neighbouring minima. To be specific, we choose

$$
V(\varphi)=\frac{1}{\ell^{2}}\left(-12+\frac{\Delta_{-}\left(4-\Delta_{-}\right)}{2} \varphi^{2}+\frac{\Delta_{-}\left(4-\Delta_{-}\right)}{4} \varphi^{4}\right)
$$

where $\ell$ is the UV AdS length. In the following, we will set $\ell=1$ to remove clutter. The potential exhibits a maximum at $\varphi_{\max }=0$ and two minima at $\varphi_{\min }= \pm 1$. In the context of holography, the maximum is associated with a UV CFT perturbed by a scalar operator of dimension $\Delta_{+}=4-\Delta_{-}$. The minima are associated with IR fixed points for flows with vanishing UV curvature, while flows for finite UV curvature end at generic points $\varphi_{0}$ with $-\left|\varphi_{\min }\right|<\varphi_{0}<\left|\varphi_{\min }\right|$.

\subsection{IR-AdS with constant $U(\varphi)$}

To complete the model we also need to specify the brane potential $W_{B}$ and the function $U$. For the first numerical study we choose

$$
W_{B}(\varphi)=\omega \exp (\gamma \varphi), \quad U(\varphi)=1
$$

with $\omega$ and $\gamma$ numerical parameters. This choice for $W_{B}$ ensures that it exhibits significant variation when the brane is displaced in $\varphi$. Furthermore, scalar fields with exponential potentials also arise frequently in effective (supergravity) theories obtained from string compactifications. In contrast, in this first example we choose $U(\varphi)$ to be unaffected with the position of the brane and hence take $U(\varphi)$ to be constant. This will be relaxed in the next numerical study in section 3.2. To be specific, the numerical parameters used in this section are given by

$$
\Delta_{-}=1.2, \quad \omega=-0.015, \quad \gamma=5
$$

Note that the phenomena we will describe do not hinge on this precise choice of parameters, which are in no way special.

Before we describe our findings, we describe scope and method of our numerical analysis. Here, all solutions will originate from the UV fixed point at the maximum $\varphi_{\max }=0$. The UV theory at the fixed point is characterised by the values of the UV curvature $R^{(\zeta)}$ and the the coupling $\varphi_{-}$of the dual theory, and we are free to adjust these parameters. The idea is to scan over these parameters, or equivalently over $\mathcal{R}=R^{(\zeta)}\left|\varphi_{-}\right|^{-2 / \Delta_{-}}$and the sign of $\varphi_{-}$. For every choice of UV sources we then check whether the flow admits a 
solution of the junction conditions at some value $\varphi_{\star}$ section 2.3 where the brane can be located. In addition, we need to make sure that on the IR side of the brane the solution is completed to a non-singular flow ending at some $\varphi_{0}$.

In practice, it is more convenient to work backwards, i.e. to begin at an IR end/turning point $\varphi_{0}$ and follow the flow backwards. The reason is that solutions that are regular in the IR only constitute a small subset compared to solutions which are singular in the IR. Hence, when starting from the UV it is numerically hard to pick out IR regular solutions. In contrast, no such problem arises when starting with a regular solution in the IR. This can typically be followed back to the UV without any problems as the UV fixed point is an attractor. Thus, our strategy is as follows:

1. We begin at some IR end/turning point $\varphi_{0}$ and solve for $W_{\mathrm{IR}}, S_{\mathrm{IR}}$ and $T_{\mathrm{IR}}$ with end or turning point at $\varphi_{0}$ with regular boundary conditions as listed in appendix C.

2. Given this solution, we then turn to the junction conditions (2.54) to search for possible brane loci $\varphi_{\star}$. Note that the junction conditions may have several solutions.

3. For every tentative brane locus $\varphi_{\star}$ we then check whether the solution can be continued on the UV side. To be acceptable, a solution on the UV side has to connect to the UV fixed point at $\varphi_{\max }=0$.

4. Once we have an acceptable solution we can then read off the UV data $\mathcal{R}$ and the sign of $\varphi_{-}$as well as the corresponding brane curvature $R_{B}=T\left(\varphi_{\star}\right)$.

5. To ensure that we capture all solutions associated with $\varphi_{\max }=0$ we employ the following strategy. By performing this analysis for any value of the end/turning point $\varphi_{0}$ in the interval $-\left|\varphi_{\min }\right|<\varphi_{0}<\left|\varphi_{\min }\right|$ we can make sure that no solutions are missed. This is equivalent to scanning over all possible values of $\mathcal{R}$ and $\operatorname{sign}\left(\varphi_{-}\right)$ in the UV.

6. Last, note that both on the UV or IR side of the brane the RG flow solutions can in principle exhibit one or multiple bounces, i.e. reversals of the flow in $\varphi$ as described at the end of section 2.2 .

Solutions with $\mathcal{R}>\mathbf{0}$. As we will see, the space of solutions is very rich. One way of organising our results is then to distinguish between solutions with positive brane (and UV) curvature and negative brane (and UV) curvature. Here we begin with the former case.

To capture all solutions we solve numerically, scanning over all possible end points $\varphi_{0}$ with $-\left|\varphi_{\min }\right|<\varphi_{0}<\left|\varphi_{\min }\right|$. For every possible end point we check for solutions and, if they exist, we record the equilibrium brane position $\varphi_{\star}$, the brane curvature $R_{B}$ and UV data (dimensionless curvature $\mathcal{R}$ and sign of the source $\varphi_{-}$).

One convenient way of presenting the space of all solutions, is to plot brane quantities $\left(\varphi_{\star}, R_{B}\right)$ vs. UV data. For example, in figure 6 we plot the brane equilibrium position $\varphi_{\star}$ vs. the dimensionless curvature $\mathcal{R}$. We can then make the following observations.

- We find that for a given value of $\mathcal{R}$ there can be up to two solutions, one with $\varphi_{-}>0$ (red) and one with $\varphi_{-}<0$ (blue). Recall that $\varphi_{-}$is the UV value of the coupling 


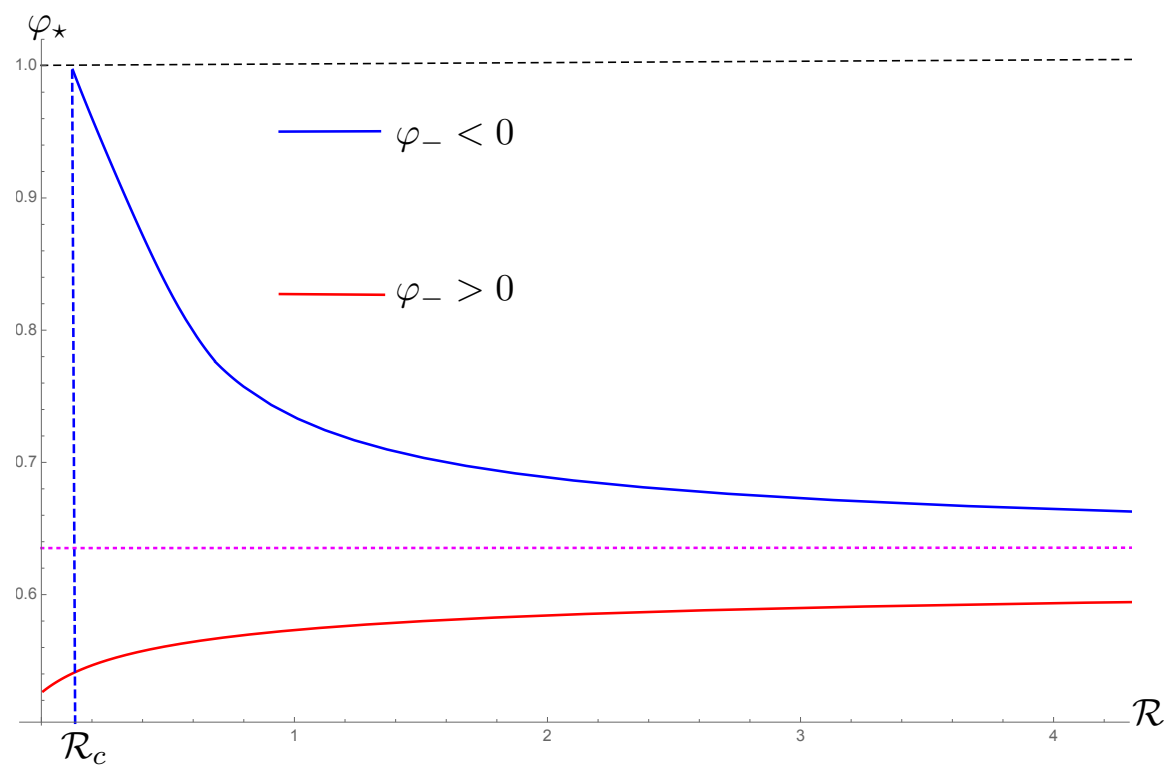

Figure 6. Equilibrium brane position $\varphi_{\star}$ vs. $\mathcal{R}$ for bulk potential (3.1), brane quantities (3.2) and parameter values (3.3). The blue line corresponds to solutions with $\varphi_{-}<0$ while the red line denotes solutions with $\varphi_{-}>0$. The brane position cannot exceed the position of the minimum of the potential at $\varphi_{\min }=1$, which is indicated by the black dashed line. For $\mathcal{R} \rightarrow \infty$ both the red and blue branch asymptote to the value for $\varphi_{\star}$ indicated by the dotted magenta line.

of the theory dual to this geometry and thus a parameter that is fixed once the dual theory is specified..

- Solutions on the red branch in figure 6 exist for all values of $\mathcal{R}>0$, i.e. there is no gap in $\mathcal{R}$. One can further check that for $\mathcal{R} \rightarrow 0$ this branch of solutions continuously connects to a solution with $\mathcal{R}=0$ and a flat brane. In contrast, solutions on the blue branch only exist for $\mathcal{R}$ larger than some particular value $\mathcal{R}_{c}$, with $\mathcal{R}_{c} \simeq 0.16$.

- We examine the solutions on the two branches in some more detail. In figure 7 we plot $W(\varphi)$ for two representative solutions with $\varphi_{-}>0$ and $\varphi_{-}<0$ corresponding to the same value of $\mathcal{R}$. In both cases (figure $7(\mathrm{a})$ and figure $7(\mathrm{~b})$ ) the brane equilibrium position is at some positive value $\varphi_{\star}$ and $W$ is discontinuous there. The main difference is that for $\varphi_{-}<0$ shown in figure 7 (b) the 'flow' leaves the UV fixed point to the left. The solution then reverses direction in $\varphi$ (i.e. it 'bounces') and continues towards positive values of $\varphi$. In the the case $\varphi_{-}>0$ shown in figure 7 (a) the flow leaves the UV to the right and no subsequent reversal of direction occurs.

- From figure 6 we can understand why solutions with $\varphi_{-}<0$ (the blue branch) stop existing for $\mathcal{R}<\mathcal{R}_{c}$. When approaching $\mathcal{R} \rightarrow \mathcal{R}_{c}$ the brane equilibrium position on the blue branch is pushed onto the minimum of the potential $\varphi_{\min }=1$. As the brane cannot move beyond the minimum this branch of solutions ends. 


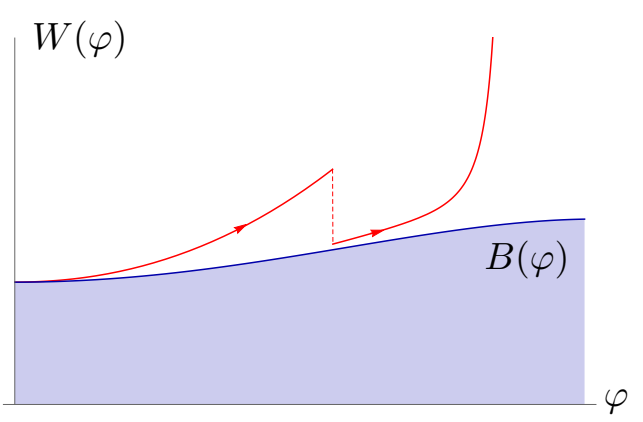

(a)

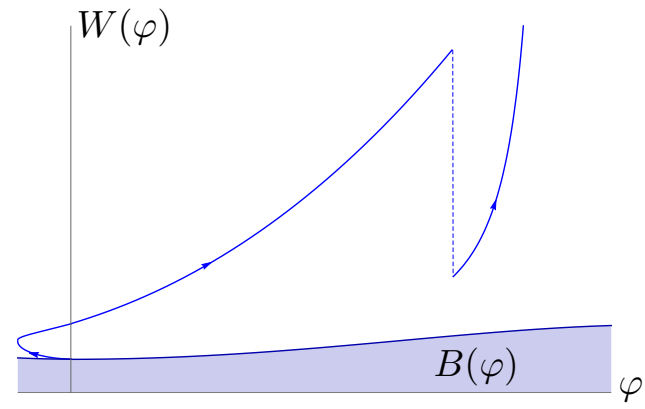

(b)

Figure 7. Two solutions for $W(\varphi)$ with the same value of $\mathcal{R}$ but different sign of $\varphi_{-}$. The results were obtained for bulk potential (3.1), brane quantities (3.2) and parameter values (3.3). The jump in $W$ is the discontinuity across the brane. In figure (a) we plot a solution with $\varphi_{-}>0$, while in (b) a solution with $\varphi_{-}<0$ is shown. Note that the solution with $\varphi_{-}<0$ exhibits a bounce, i.e. a reversal of direction in $\varphi$.

- We now return to the discussion of the full space of solutions in figure 6. Another observation is that the equilibrium position for the brane over the whole solution space is limited to a particular band in $\varphi$. Here we find that the brane can only be located in the range $\varphi_{\star, 0} \leq \varphi_{\star}<\left|\varphi_{\min }\right|$, with $\varphi_{\star, 0} \simeq 0.525$. For example, this implies that for this model the brane can never sit at values $\varphi_{\star}<0$, even though we did not exclude this in the analysis.

- Finally, note that the two branches of solutions $\left(\varphi_{-}>0\right.$ and $\left.\varphi_{-}<0\right)$ are connected. However, to go from $\varphi_{-}>0$ to $\varphi_{-}<0$ the source has to cross $\varphi_{-}=0$ and the dimensionless curvature $\mathcal{R}=R^{(\zeta)}\left|\varphi_{-}\right|^{-2 / \Delta_{-}}$diverges. Thus, the point where the two branches of solutions meet is displaced to $\mathcal{R} \rightarrow \infty$ in figure 6 . The corresponding value of $\varphi_{\star}$ is indicated by the dotted magenta line. The solution with $\varphi_{-}=0$ corresponds to a vev flow in the gravity dual language, as the RG flow is induced not by the source $\varphi_{-}$of an operator $\mathcal{O}$, but by its vev $\langle\mathcal{O}\rangle$. This solution has with a fixed value of $\chi \equiv R^{(\zeta)}|\langle\mathcal{O}\rangle|^{-2 / \Delta_{+}}$. Thus, it is actually a one-parameter space of solutions spanned by all values of $R^{(\zeta)}$ and $\langle\mathcal{O}\rangle$ such that $\chi$ remains constant. For more details we refer readers to [33]. A similar situation has been extensively discussed in [56] for thermal states in holographic theories, where the role of curvature is taken by temperature.

Having discussed the space of solutions, we will now describe how the brane curvature $R_{B}$ varies across the solution space. In particular, for 4-dimensional observers the main quantity of interest is brane curvature $R_{B}$ in units of the 4-dimensional Planck scale $M_{4}$. This is defined as

$$
M_{4}^{2} \equiv M_{P}^{2} U_{B}\left(\varphi_{\star}\right)
$$

where $M_{P}$ is the $5 \mathrm{~d}$ Planck scale as introduced in (2.2) and (2.3). Thus, in figure 8 where we display $R_{B} M_{4}^{-2}$ vs. $\mathcal{R}$. We again use the colours blue and red to distinguish between 


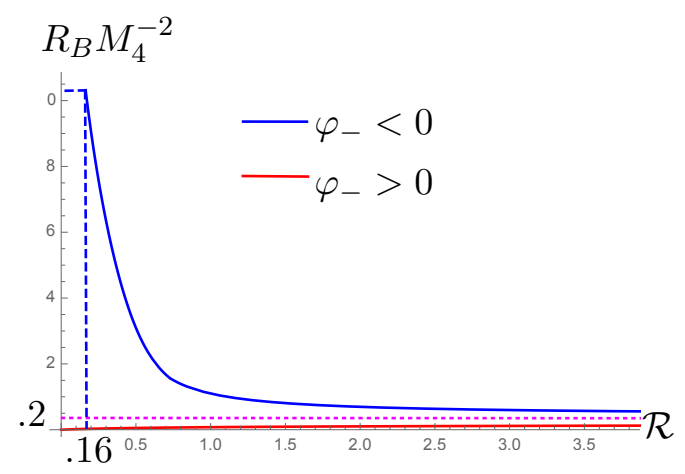

(a)

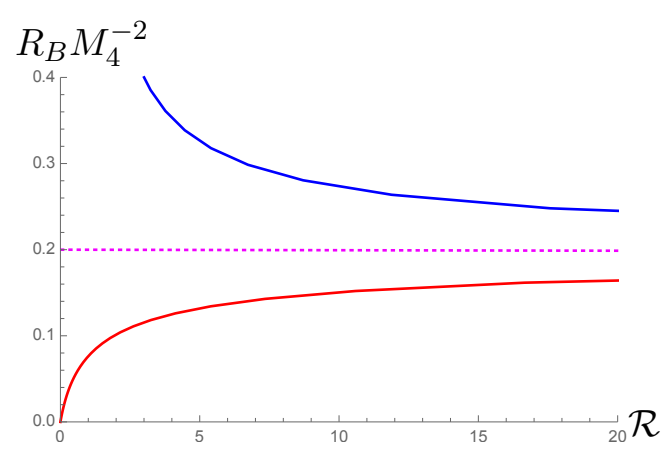

(b)

Figure 8. Brane curvature in units of the $4 \mathrm{~d}$ Planck mass $R_{B} M_{4}^{-2}$ vs. $\mathcal{R}$ for bulk potential (3.1), brane quantities (3.2) and parameter values (3.3). In figure (a) both solutions with $\varphi_{-}<0$ and $\varphi_{-}>0$ are shown. In figure (b) we adjusted the plot range to improve visibility of the result with $\varphi_{-}>0$. For $\mathcal{R} \rightarrow \infty$ both the red and blue branch asymptote to the value for $R_{B} M_{4}^{-2}$ indicated by the dotted magenta line.

the two branches of solutions with $\varphi_{-}<0$ and $\varphi_{-}>0$ respectively. We make the following observations.

- We begin by describing the branch of solutions with $\varphi_{-}>0$, which is seen most clearly in figure $8(\mathrm{~b})$. This branch of solutions exists for all values of $\mathcal{R}$. Note that for $\mathcal{R} \rightarrow 0$ we find $R_{B} \rightarrow 0$. Thus, in this limit we recover a solution with a flat brane in a setup with no UV curvature, a configuration studied before in [20]. The brane curvature $R_{B}$ then grows monotonically with $\mathcal{R}$. For $\mathcal{R} \rightarrow \infty$ the brane curvature then asymptotes to the value $R_{B, 0} M_{4}^{-2} \simeq 0.2$ from below.

- The branch of solutions with $\varphi_{-}<0$ is depicted most clearly in figure 8 (a). Recall that solutions of this type only exist for $\mathcal{R} \geq \mathcal{R}_{c} \simeq 0.16$. At this point the brane curvature takes the maximum value $R_{B, \max } M_{4}^{-2} \simeq 10.6$. It then falls monotonically with increasing $\mathcal{R}$, asymptoting to $R_{B, 0} M_{4}^{-2} \simeq 0.2$ from above for $\mathcal{R} \rightarrow \infty$.

In the following, we will also quantify to what extent the embedding of the brane in the bulk modifies the value for $R_{B}$ compared to the value expected from the $4 \mathrm{~d}$ cosmological constant alone. In particular, a brane at position $\varphi_{\star}$ exhibits a cosmological constant $W_{B}\left(\varphi_{\star}\right)$. If $4 \mathrm{~d}$ gravity was the only physics governing the geometry of the brane, this would result in a brane curvature

$$
R_{\star}=2 \frac{W_{B}\left(\varphi_{\star}\right)}{U\left(\varphi_{\star}\right)}
$$

Hence, in figure 9 we plot $R_{B} /\left|R_{\star}\right|$ vs. $\mathcal{R}$. We put an absolute value sign as $R_{\star}<0$ in this example. We make the following observations. Generically one finds $R_{B} /\left|R_{\star}\right| \sim \mathcal{O}(1)$. That is, while the presence of the bulk modifies the value of $R_{B}$ somewhat, it is is no way 'tuned' small generically. The only regime where $R_{B} /\left|R_{\star}\right| \ll 1$ is on the red branch when $\mathcal{R} \rightarrow 0$. That is, we find that $R_{B} /\left|R_{\star}\right|$ is small exactly when $R_{B} M_{4}^{-2}$ is also small, as 


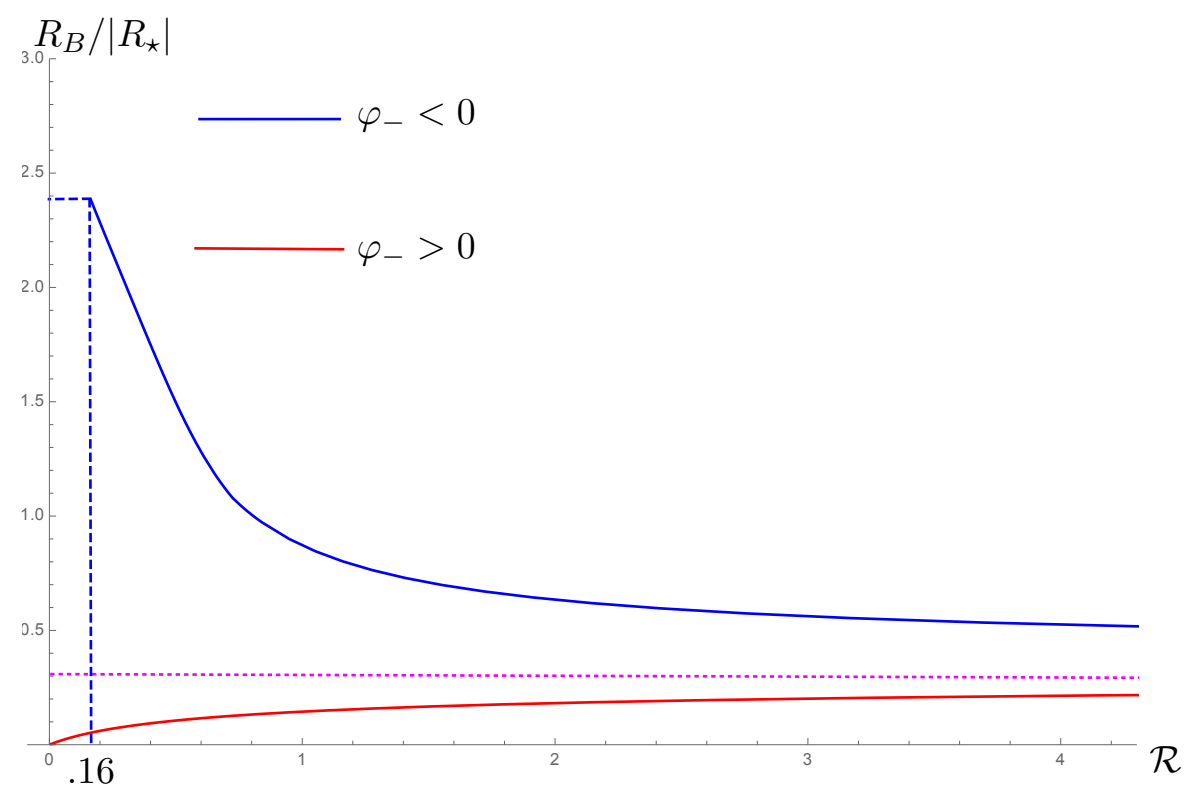

Figure 9. $R_{B} /\left|R_{\star}\right|$ as a function of $\mathcal{R}$ for bulk potential (3.1), brane quantities (3.2) and parameter values (3.3). $\left|R_{\star}\right|$ is defined as the 'expected' brane curvature related to the brane cosmological constant $W_{B}\left(\varphi_{\star}\right)$ in the absence of a $5 \mathrm{~d}$ bulk. For $\mathcal{R} \rightarrow \infty$ both the red and blue branch asymptote to the value for $R_{B} /\left|R_{\star}\right|$ indicated by the dotted magenta line.

can be seen by comparing with figure 8 . We will make the same qualitative observations for $R_{B} /\left|R_{\star}\right|$ vs. $\mathcal{R}$ for all following models in this work. Hence we refrain from plotting $R_{B} /\left|R_{\star}\right|$ vs. $\mathcal{R}$ in the remainder of this work.

Before moving on to solutions with $\mathcal{R}<0$, we summarise the main findings. There are four points to be taken away from studying this example:

1. The range of possible values that $R_{B}$ can take is bounded in the model studied here. In particular, we find that $R_{B}$ is constrained to lie in the interval $0 \leq R_{B}<R_{B \text {, max }}$.

2. The solution with $R_{B}=0$ also exhibits $\mathcal{R}=0$. Thus, in this model a flat brane is only obtained if the UV curvature also vanishes and vice versa.

3. We observe that a hierarchically low brane curvature $R_{B} M_{4}^{-2} \ll 1$ only occurs in the vicinity of the flat solution. More precisely, we only find solutions with $R_{B} M_{4}^{-2} \ll 1$ if we also choose $\mathcal{R} \ll 1$. For generic values of $\mathcal{R}$ we find that $R_{B} M_{4}^{-2} \sim \mathcal{O}(1)$.

4. Most interestingly, we find that even when the UV curvature diverges, $\mathcal{R} \rightarrow \infty$, the brane curvature stays finite.

Solutions with $\mathcal{R}<\mathbf{0}$. The space of solutions with negative UV curvature is even richer than in the positive curvature case. We plot $\varphi_{\star}$ vs. $\mathcal{R}$ for $\mathcal{R}<0$ in figure 10 . We see that for a given value of $\mathcal{R}$ up to six solutions exist, three of which exhibit $\varphi_{-}<0$ (denoted in blue) and three $\varphi_{-}>0$ (red). Note that branch denoted by the dashed red line only 


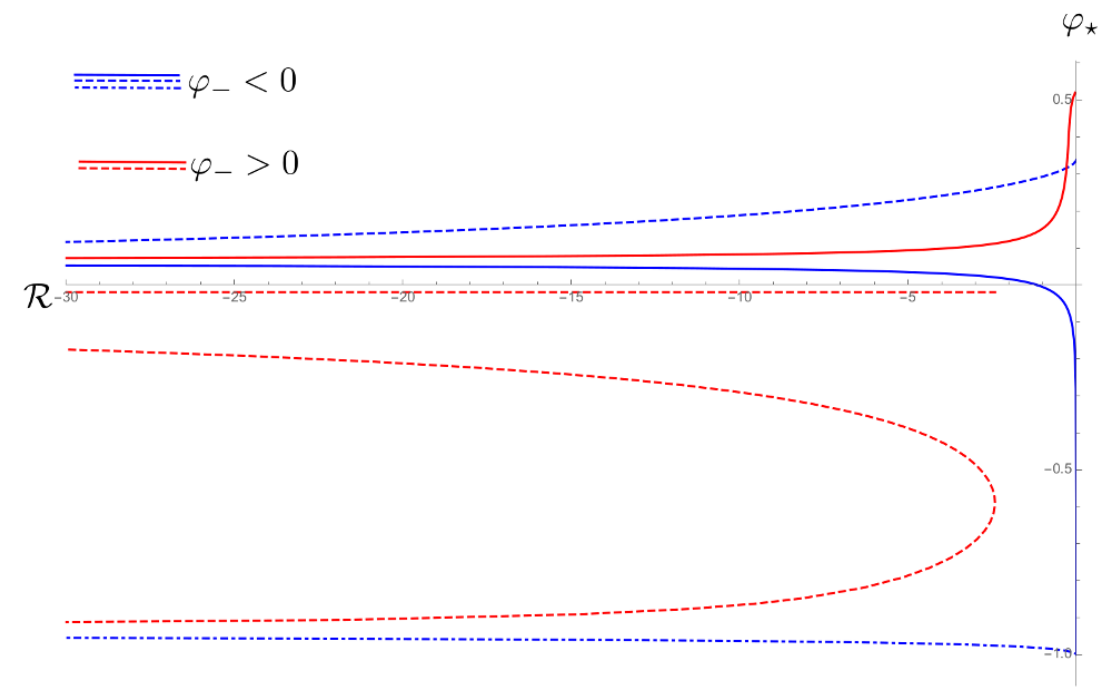

Figure 10. Equilibrium brane position $\varphi_{\star}$ vs. $\mathcal{R}$ for $\mathcal{R}<0$. Results are obtained for bulk potential (3.1), brane quantities (3.2) and parameter values (3.3). Blue lines corresponds to solutions with $\varphi_{-}<0$ while red line denotes solutions with $\varphi_{-}>0$.

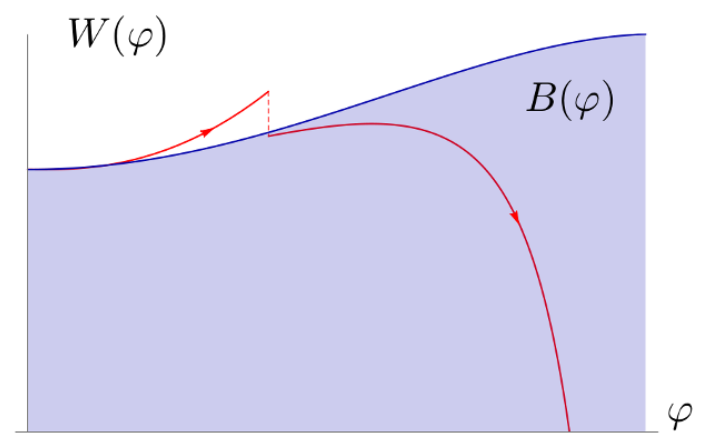

(a)

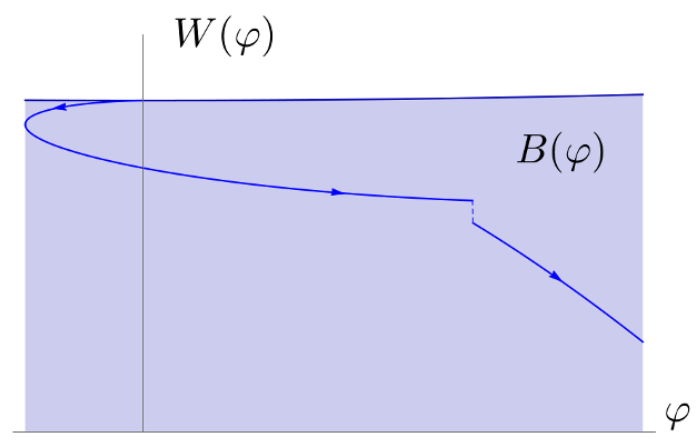

(b)

Figure 11. Two solutions for $W(\varphi)$ with the same (negative) value of $\mathcal{R}$ but different $\operatorname{sign}$ of $\varphi_{-}$. The results were obtained for bulk potential (3.1), brane quantities (3.2) and parameter values (3.3). The jump in $W$ is the discontinuity across the brane. In figure (a) we plot a solution with $\varphi_{-}>0$, while in (b) a solution with $\varphi_{-}<0$ is shown. Note that the solution with $\varphi_{-}<0$ exhibits a bounce, i.e. a reversal of direction in $\varphi$.

exists when the UV curvature is sufficiently negative, i.e. $\mathcal{R}<\mathcal{R}_{c, 2}$ with $\mathcal{R}_{c, 2} \simeq-3.5$. The other branches exist for all $\mathcal{R}<0$.

We display example solutions for $W(\varphi)$ with $\mathcal{R}<0$ in figures 11. For one, we find solutions without a bounce, i.e. without a change of direction, as shown in figure 11(a). This is observed, for example, on the solid red branch in figure 10. Also note that for $\mathcal{R}<0$ we have $W=0$ in the IR. A representative solution for $W(\varphi)$ with a bounce is shown in figure 11(a). This occurs, for example, on the solid blue branch with $\varphi_{\star}>0$. 
Before moving on, we will have a closer look at the four branches in figure 10 that admit a limit $\mathcal{R} \rightarrow 0$ :

- We find that the solid red branch is continuously connected to a solution with $\mathcal{R}=0$ exactly. The brane position approaches and finally coincides with that of a flat brane when letting $\mathcal{R} \rightarrow 0$.

- For the solid blue branch the brane is pushed towards the minimum of the potential at $\varphi_{\min }=-1$ when letting $\mathcal{R} \rightarrow 0$. While it can be arbitrarily close to $\varphi_{\min }$, it can never coincide with it. The reason is that for $\varphi \rightarrow \varphi_{\min }$ the bulk geometry shrinks to a point. Thus the strict limit $\varphi_{\star} \rightarrow \varphi_{\min }$ is equivalent to the absence of the brane.

- A more interesting phenomenon is observed for $\mathcal{R}$ for the solutions described by the dashed and dot-dashed blue lines in figure 10. This is best seen by plotting $W(\varphi)$ for the dashed blue branch for $\mathcal{R} \rightarrow 0$. This is shown in figure 12. Note that this solution exhibits a bounce, and for $\mathcal{R} \rightarrow 0$ the location of the bounce approaches the minimum of the potential at $\varphi_{\min }=-1$. We can then understand why the solution does not exist in the strict limit $\mathcal{R}=0$. In this case the flow leaving the UV fixed point at $\varphi_{\max }=0$ passes through $\varphi_{\min }$ exactly, which becomes the IR end point for the flow. The geometry shrinks to a point there and the flow ends before reaching the brane, thus excluding a solution with $\mathcal{R}=0 .{ }^{14}$

To summarise, while we have many solutions that permit arbitrarily small $\mathcal{R}$, only the branch denoted by the solid red line is continuously connected to a solution with $\mathcal{R}=0$.

We briefly return to figure 12 and make another comment. Note that this solution exhibits a region with $W<0$ and hence $W$ changes sign. As $W \sim \dot{A}$ this implies that $A$ is not monotonic in $u$. In the case of holographic RG flows such solutions would be excluded. The reason is that $A$ or rather $e^{A}$ is interpreted as the RG scale, which should be monotonic along the flow. Here we do not rely on the interpretation in terms of RG flows and hence we see no reason to exclude such solutions.

We now examine the brane curvature $R_{B} M_{4}^{-2}$ in 4 d Planck units. In particular, in figure 13(b) we plot $R_{B} M_{4}^{-2}$ vs. $\mathcal{R}$ for solutions with $\varphi_{\star}>0$. For better visibility, we split the plot into two, covering different ranges in $R_{B} M_{4}^{-2}$. We make the following observations:

- Of all the branches of solutions, only the solid red and blue branches admit solutions with hierarchically small $R_{B}$, i.e. they exhibit $R_{B} \rightarrow 0$ for $\mathcal{R} \rightarrow 0$. However, as remarked before, only the solid red branch connects continuously to a solution with $R_{B}=0$. On the solid blue branch the strict limit $R_{B}=0$ cannot be reached. All other solutions in this model exhibit $R_{B} M_{4}^{-2} \sim \mathcal{O}(1)$.

- For all the branches of solutions we find that $R_{B} M_{4}^{-2}$ stays finite as $|\mathcal{R}| \rightarrow \infty$. This is the same qualitative behaviour as observed for $\mathcal{R}>0$ before.

\footnotetext{
${ }^{14}$ The remaining part of the flow, i.e. the part beyond $\varphi_{\min }=-1$ can then be understood as a solution where now the minimum at $\varphi_{\text {min }}=-1$ plays the role of UV fixed point. Hence it is not part of the space of solutions associated UV fixed point $\varphi_{\max }=0$.
} 


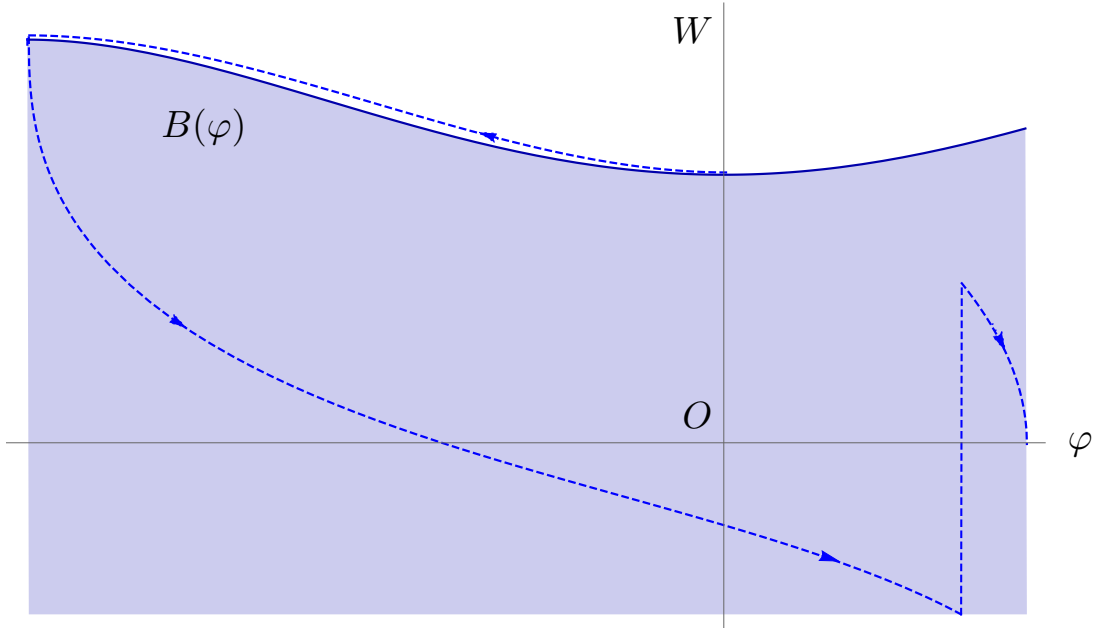

Figure 12. $W(\varphi)$ for $\mathcal{R}<0$. The results were obtained for bulk potential (3.1), brane quantities (3.2) and parameter values (3.3). This is a solution of the branch of solutions denoted by the dashed blue line in 10 with $\mathcal{R} \rightarrow 0$. The solution exhibits a reversal of direction, and for $\mathcal{R} \rightarrow 0$ the bounce locus is pushed towards a minimum of the potential. Note that this solution also exhibits a region with $W<0$.

$\mathcal{R}$

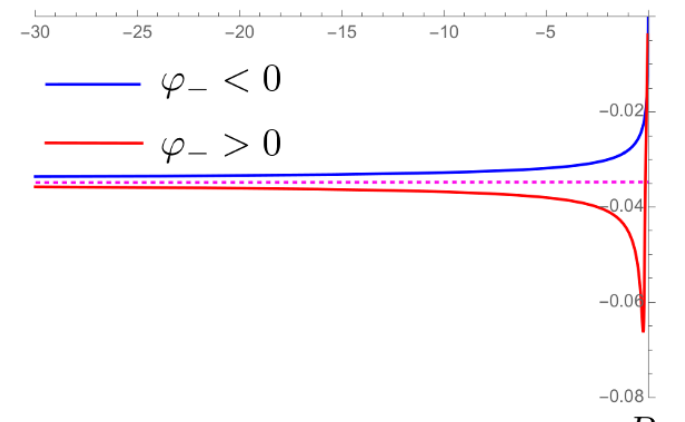

(a)
$\mathcal{R}$

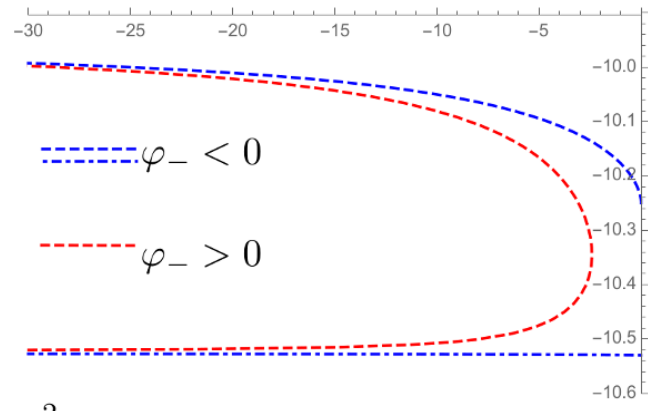

(b)
$R_{B} M_{4}^{-2}$

Figure 13. Brane curvature in $4 \mathrm{~d}$ Planck units $R_{B} M_{4}^{-2}$ vs. $\mathcal{R}$ with $\mathcal{R}<0$ for bulk potential (3.1), brane quantities (3.2) and parameter values (3.3). In figure (a) we show results corresponding to the branches denoted by solid lines in figure 10. In figure (b) the results for the dashed and dot-dashed branches in figure 10 are displayed.

- We again find something new for the dashed and dot-dashed blue branches. Here we observe that $R_{B} M_{4}^{-2}$ stays finite for $\mathcal{R} \rightarrow 0$. This can be understood by looking again at figure 12. While the bounce locus is pushed towards a minimum for $\mathcal{R} \rightarrow 0$, the brane remains located at a generic point thus exhibiting finite curvature.

- Finally note that $R_{B}$ is bounded, i.e. we cannot obtain solutions with $R_{B} M_{4}^{-2}<$ $-10.25$ 
Summary. This concludes our description of the full space of solutions of our bulkbrane system for the model described by the bulk potential (3.1), brane quantities (3.2) and parameter values (3.3). We have made four main qualitative observations.

1. Our setup allows for solutions with a flat brane, i.e. with $R_{B}=0$. However, we find that these can only be obtained if the UV curvature is also chosen to vanish, i.e. $\mathcal{R}=0$. These are the flat self-tuning solutions of [20].

2. Solutions with a hierarchically small $R_{B}$ (that is $R_{B} M_{4}^{-2} \ll 1$ ) exist, but require $\mathcal{R} \ll 1$ to be tuned small.

3. At the same time, solutions exist with finite but arbitrarily small $\mathcal{R}$ that nevertheless exhibit a finite $R_{B} M_{4}^{-2}$.

4. While solutions with positive and negative $R_{B}$ exist, the brane curvature is bounded. In particular, solutions populate an interval $-\left|R_{B, 1}\right|<R_{B}<\left|R_{B, 2}\right|$.

5. Finally, we observe that the brane curvature $R_{B}$ remains finite even if $\mathcal{R} \rightarrow \infty$. Thus large hierarchies between $R_{B} M_{4}^{-2}$ and $\mathcal{R}$ can be achieved.

There is a further caveat regarding the solutions in this section. In particular, we should also check explicitly, whether the solution portrayed here satisfy the stability criteria listed in (2.56)-(2.59). One finds that our choice $W_{B}<0$ is in conflict with criterion (2.57), which in practice often reduces to the requirement $W_{B}\left(\varphi_{\star}\right)>0$. However, note that (2.57) is only a sufficient condition and its violation thus does not automatically imply an instability. To check stability of our solutions would thus require a more detailed analysis, which goes beyond the scope of this paper. However, in section 4 we will consider a different model based on different choices for $V(\varphi), W_{B}(\varphi)$ and $U(\varphi)$, which will exhibit $W_{B}\left(\varphi_{\star}\right)>0$.

\section{$3.2 \quad$ IR-AdS with exponential $U(\varphi)$}

So far we have considered a model with $U(\varphi)=1=$ const. In this section we will depart from this choice in a drastic way. In particular, we now choose $U(\varphi)$ to be exponentially sensitive to the brane position. Thus we will now consider a model with bulk potential (3.1) and brane quantities given by

$$
W_{B}(\varphi)=\omega \exp (\gamma \varphi), \quad U(\varphi)=|\omega| \exp (\gamma \varphi),
$$

with

$$
\Delta_{-}=1.2, \quad \omega=-0.015, \quad \gamma=5 .
$$

To simplify the analysis we have chosen both $W_{B}(\varphi)$ and $U(\varphi)$ to be described by the same exponential function. ${ }^{15}$

We will now explore the space of solutions of our bulk-brane system for this model. As before, we will distinguish between setups with positive and negative UV curvature.

\footnotetext{
${ }^{15}$ Furthermore, we expect $\varphi$-dependence of the brane potential $W_{B}(\varphi)$ and of $U(\varphi)$ to have a common physical origin. Thus choosing related functions for $W_{B}$ and $U$ may not be unrealistic.
} 


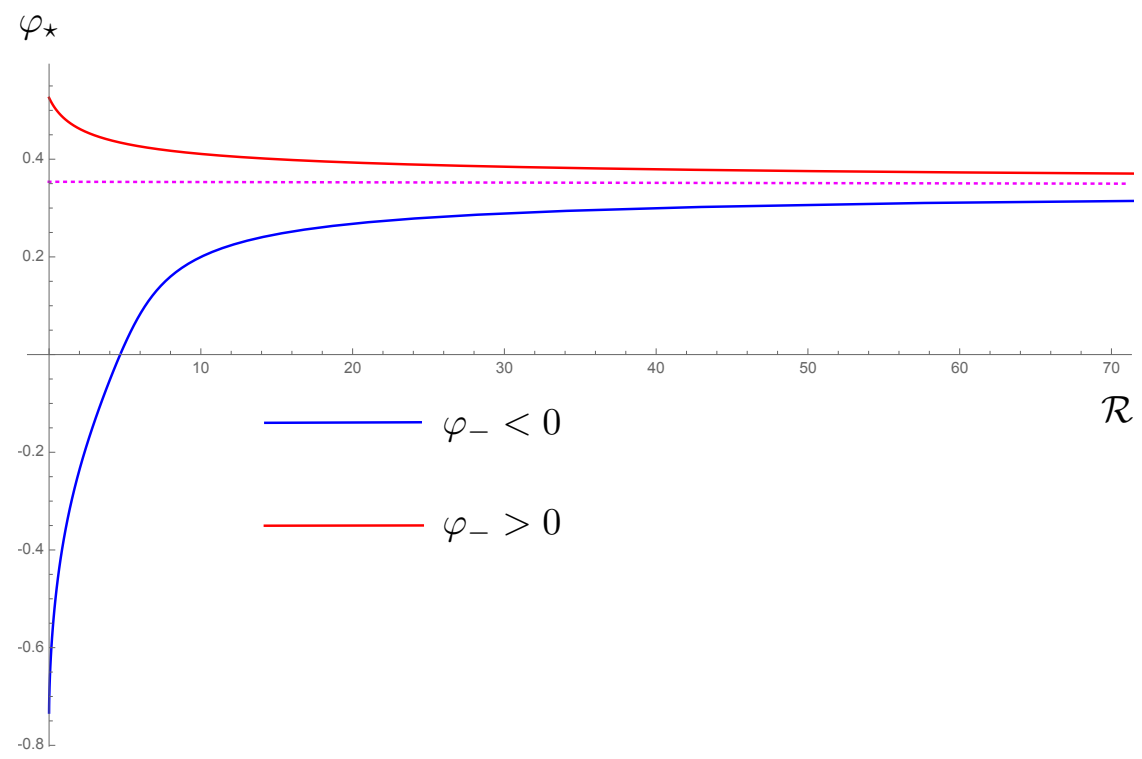

Figure 14. Equilibrium brane position $\varphi_{\star}$ vs. $\mathcal{R}$ for $\mathcal{R}>0$. Results are obtained for bulk potential (3.1), brane quantities (3.6) and parameter values (3.7). For $\mathcal{R} \rightarrow \infty$ both the red and blue branch asymptote to the value for $\varphi_{\star}$ indicated by the dotted magenta line.

Solutions with $\mathcal{R}>\mathbf{0}$. We display this part of the space of solutions by plotting $\varphi_{\star}$ vs. $\mathcal{R}$ which is shown in figure 14. We find that for a given value of $\mathcal{R}$ there are up to two branches of solutions, one with $\varphi_{-}>0$ (red) and one with $\varphi_{-}<0$ (blue). This is similar to the case with $U(\varphi)=1$ (see figure 6) studied before. However, we find the following differences to the model $U(\varphi)=1$. For one, here both branches of solutions exist for arbitrarily small $\mathcal{R}$. The most important difference is that there are now solutions with $\varphi_{\star}<0$ while such solutions were absent for $U(\varphi)=1$. The remaining differences are just quantitative, i.e. how $\varphi_{\star}$ precisely behaves as a function of $\mathcal{R}$.

In figure 15 we plot $R_{B} M_{4}^{-2}$ as a function of $\mathcal{R}$ for the model with exponential $U(\varphi)$. This is to be compared with the corresponding findings for the model with $U(\varphi)=1$ which are plotted in figure 8 . Here, while there are numerical differences between the two models, they exhibit similar qualitative results. Again, we find that $R_{B} \rightarrow 0$ can only occur when simultaneously $\mathcal{R} \rightarrow 0$. However, here the brane curvature $R_{B}$ is not necessarily bounded. The value of $R_{B} M_{4}^{-2}$ diverges on the blue branch for $\mathcal{R} \rightarrow 0$.

Solutions with $\mathcal{R}<\mathbf{0}$. We also find solutions with $\mathcal{R}<0$. In fact, here the space of solutions with negative UV curvature is even more involved than in the case with constant $U(\varphi)$. However, the picture that emerges is similar to what we have seen for $\mathcal{R}>0$ : while the results change quantitatively, the main qualitative observations persist. For example, we again observe that solutions with arbitrarily small $|\mathcal{R}|$ but finite $R_{B} M_{4}^{-2}$. As we do not find any great qualitative differences we refrain from showing any explicit results. 


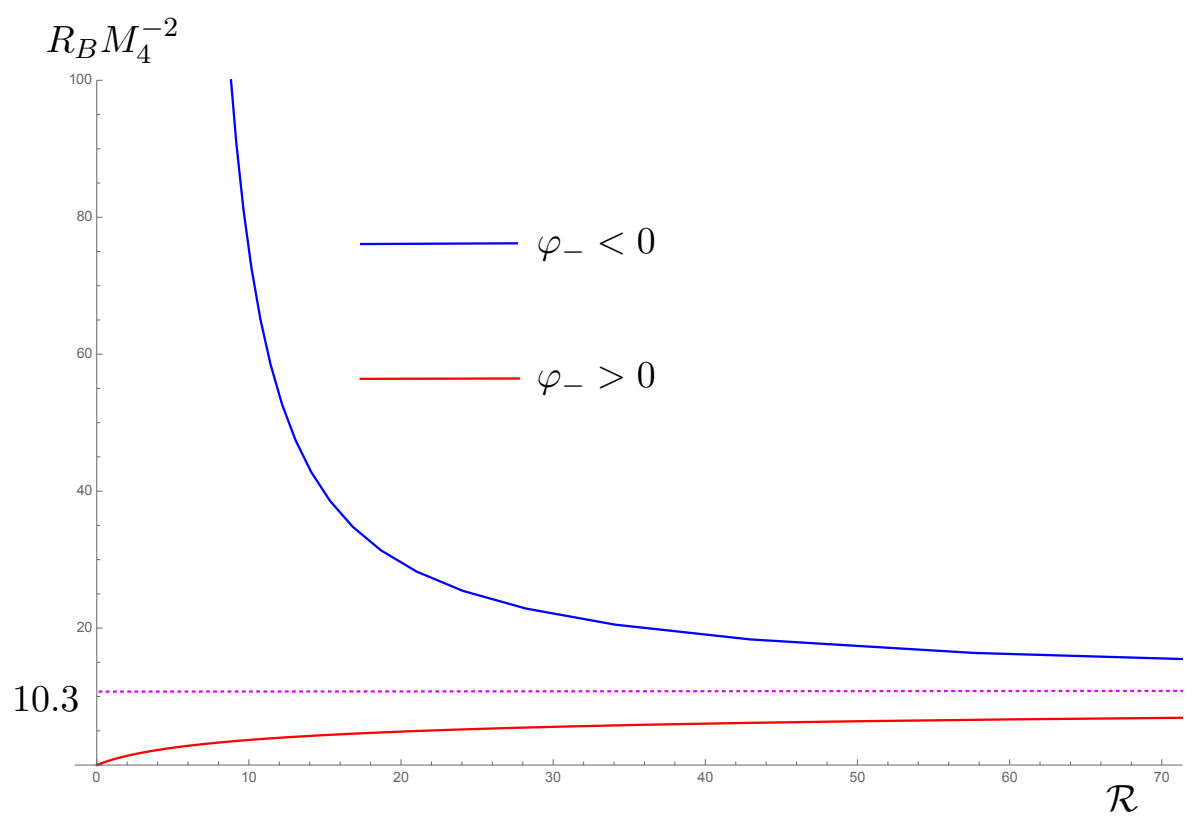

Figure 15. Brane curvature $R_{B} M_{4}^{-2}$ vs. $\mathcal{R}$ for $\mathcal{R}>0$. Results are obtained for bulk potential (3.1), brane quantities (3.6) and parameter values (3.7). For $\mathcal{R} \rightarrow \infty$ both the red and blue branch asymptote to the value for $R_{B} M_{4}^{-2}$ indicated by the dotted magenta line.

\section{IR exponential potential}

In the previous section, we studied self-stabilisation in models with a finite range in $\varphi$ between the UV and IR. This constraint arose from choosing a bulk potential with one maximum surrounded by two minima. Solutions were confined to the region between the two minima. Here we will consider a model without this constraint and where a priori $\varphi$ has infinite range. This can be done by choosing a bulk potential with a maximum, but no minima. To be specific, we choose:

$$
V(\varphi)=\frac{1}{\ell^{2}}\left[-12-\varphi^{2}\left(\frac{1}{2} \Delta(4-\Delta)-\frac{b^{2}}{4}\right)-V_{1} \sinh ^{2}\left(\frac{b \varphi}{2}\right)\right]
$$

with $2<\Delta<4$ and $V_{1}>0$ and $b$ another (dimensionless) parameter. Note that we still work in $d=4$. We will also again set $\ell=1$ in the following. The maximum of $V$ is at $\varphi_{\max }=0$, which, in the language of holographic RG flows, is a UV fixed point with corresponding UV CFT. In this framework the parameter $\Delta$ is interpreted as the dimension of the operator perturbing this UV CFT. However, as there are no minima the IR is only reached for $|\varphi| \rightarrow \infty$. Solutions that flow all the way to $|\varphi| \rightarrow \infty$ are singular, but, as briefly reviewed in section 2.2 , they can be acceptable if certain conditions are satisfied.

One reason for choosing a potential with unlimited range in $\varphi$ is as follows. In [20], for the case of a flat brane, it was observed that bulk potentials with a finite range for $\varphi$ do not easily exhibit self-tuning solutions satisfying the stability criterion (2.57), at least not without some fine-tuning of parameters. However, for potentials with infinite range in 
$\varphi$ this difficulty can be overcome, as was shown for the case of a flat brane in [20] using an example based on bulk potential (4.1).

To allow comparisons with the results in [20] we choose a brane potential with the same mathematical form. Hence we will work with the following brane quantities:

$$
W_{B}(\varphi)=\Lambda^{4}\left[-1-\frac{\varphi}{s}+\left(\frac{\varphi}{s}\right)^{2}\right], \quad U(\varphi)=\text { const },
$$

where $\Lambda$ and $s$ are numerical parameters (as we have set $\ell=1$ ). The brane potential is chosen such that it has at least one zero for $\varphi>0$. The position of the zero is controlled by $s$. Again, absent any prior knowledge regarding $U(\varphi)$ we take it to be constant for simplicity.

\subsection{Analytical results}

Before moving on to numerical studies, we collect analytical results for the asymptotic region $\varphi \rightarrow \infty$. Note that apart from a region in the vicinity of $\varphi_{\max }=0$ the potential is well-approximated by an exponential. As a result, an analytical understanding of solutions for an exponential potential will turn out to be very helpful for the interpretation of our numerical findings.

Therefore, we collect analytical solutions for an exactly exponential bulk potential. To be specific, we will consider

$$
V=-V_{\infty} \exp (b \varphi)
$$

which is the asymptotic form of (4.1) for $\varphi \rightarrow \infty$ if we identify $V_{\infty}=V_{1} / 4$. For $\varphi \rightarrow \infty$ the bulk solutions fall into three classes.

1. Continuous branch:

For one there exists a family of solutions of the form

$$
W \simeq W_{0} e^{Q \varphi}, \quad S \simeq W^{\prime}, \quad T \simeq T_{0} e^{Q \varphi}, \quad \text { where } \quad Q=\sqrt{\frac{2}{3}} .
$$

In this case $W_{0}$ and $T_{0}$ are free parameters. The existence of this branch of solutions requires

$$
b<2 Q=2 \sqrt{\frac{2}{3}} .
$$

This branch also exists for flat solutions (albeit with $T_{0}=0$ ) and was already discussed in subsection 2.2. For $\varphi \rightarrow \infty$ these solutions exhibit an unacceptable singularity according to Gubser's criterion [25]. For more details on this class of solutions see e.g. [50].

2. A special solution with $S=W^{\prime}$ :

There exists an isolated solution of the form

$$
W=W_{0} e^{b \varphi / 2}, \quad S=W^{\prime}, \quad T=0, \quad \text { with } \quad W_{0}=\sqrt{\frac{8 V_{\infty}}{4 Q^{2}-b^{2}}},
$$


with $Q$ defined as in (4.4). Again, this solution only exists for

$$
b<2 Q=2 \sqrt{\frac{2}{3}} .
$$

This is the special flat solution satisfying Gubser's criterion, giving rise to an acceptable IR singularity, as discussed in section 2.2.

3. A special solution with $S=W /(3 b)$ :

Finally, the equations of motion (2.24)-(2.26) also admit the solution

$$
\begin{aligned}
W & =W_{0} e^{b \varphi / 2}, \quad S=\frac{W}{3 b}, \quad T=T_{0} e^{b \varphi}, \\
\text { with } \quad W_{0} & =\sqrt{6 V_{\infty}}, \quad T_{0}=\left[2 b^{2}-\frac{4}{3}\right] V_{\infty} .
\end{aligned}
$$

This solution exists for any value of $b$, but we observe that the sign of the function $T$ depends on $b$ as follows:

$$
\begin{aligned}
& b>\sqrt{\frac{2}{3}} \quad \Leftrightarrow \quad T>0, \\
& b<\sqrt{\frac{2}{3}} \quad \Leftrightarrow \quad T<0, \\
& b=\sqrt{\frac{2}{3}} \quad \Leftrightarrow \quad T=0 .
\end{aligned}
$$

For $b^{2}=2 / 3$ the solution of type 3 discussed and the one of type 2 discussed above are identical.

Here we see that non-zero curvature gives rise to a new solution reaching the asymptotic IR region $\varphi \rightarrow+\infty$, for which $W(\varphi)$ has the same exponential growth but different overall magnitude as the special, flat solution, and for which $S \neq W^{\prime}$. Depending on the value of $b$ these solution are found either for $R>0$ only, or for $R<0$ only.

Intriguingly, the critical value separating these cases, $b=\sqrt{2 / 3}$, is the same which separates confining from non-confining theories. This may signal interesting consequences in regards to confining holographic theories on curved manifolds, whose analysis we leave for further investigation.

\subsection{Numerical studies}

We now return to a study of the model with the full bulk potential (4.1) and brane quantities (4.2). In particular, we now solve numerically for solutions of the bulk-brane system. For definiteness, we will choose the following values for the parameters:

$$
\Delta=2.9, \quad \Lambda=3, \quad s=8, \quad V_{1}=1, \quad U=10^{-4},
$$




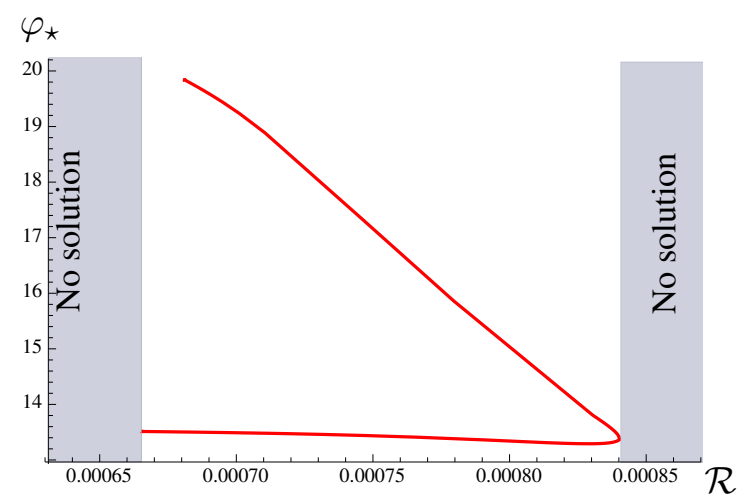

(a)

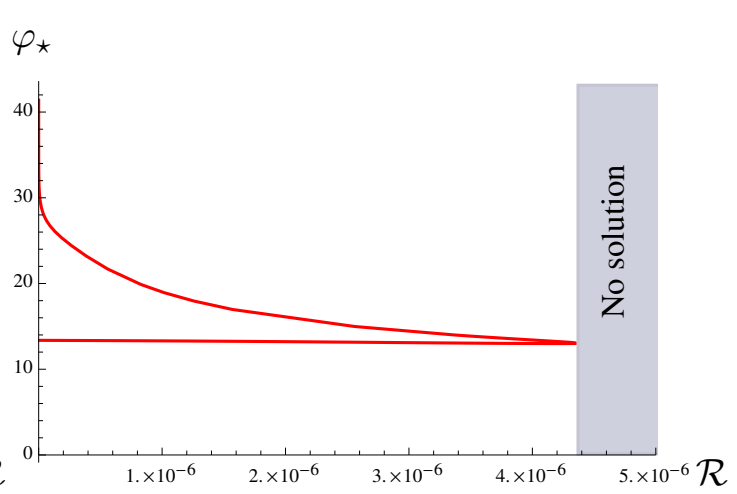

(b)

Figure 16. Equilibrium brane position $\varphi_{\star}$ vs. $\mathcal{R}$ for $\mathcal{R}>0$. Results are obtained for bulk potential (4.1), brane quantities (4.2) and parameter values (4.9). The left figure (a) is for $b=$ $1.1 \times \sqrt{2 / 3}$ and the right figure (b) is for $b=0.9 \times \sqrt{2 / 3}$.

but these values are in no way special. However, a small numerical value for $U$ will turn out to be favourable for satisfying the stability criteria (2.57) and (2.58). We then perform the analysis for two different values of $b$. In particular, we will study the cases

$$
b=1.1 \sqrt{2 / 3} \text { and } b=0.9 \sqrt{2 / 3}
$$

We will restrict our analysis to solutions with positive UV curvature $\mathcal{R}>0$, as this will exhibit all the phenomena that we wish to illustrate with this example. In figure 16 we show the space of solutions by plotting the equilibrium brane position $\varphi_{\star}$ vs. $\mathcal{R}$. In figure 16(a) we show the results for $b=1.1 \sqrt{2 / 3}$ while the results for $b=0.9 \sqrt{2 / 3}$ are displayed in figure 16(b).

One common observation for both values of $b$ is that solutions only exist for a very narrow range in $\mathcal{R}$. In particular, we find that solutions only exist for the following values of $\mathcal{R}$ :

$$
\begin{array}{ll}
b=1.1 \sqrt{2 / 3}: & \text { solutions exist for } \quad 0.00066 \lesssim \mathcal{R} \lesssim 0.00084 \text { and } \mathcal{R}=0, \\
b=0.9 \sqrt{2 / 3}: & \text { solutions exist for } \quad 0 \leq \mathcal{R} \lesssim 4.5 \cdot 10^{-6}
\end{array}
$$

That is, for $b=0.9 \sqrt{2 / 3}$ we only find solutions with very small absolute values of $\mathcal{R}$. For $b=1.1 \sqrt{2 / 3}$ solutions with finite $\mathcal{R}$ can only exist in a very narrow band of width $\Delta \mathcal{R} \sim 2 \cdot 10^{-4}$ about the central value $\mathcal{R} \sim 7.5 \cdot 10^{-4} .{ }^{16}$

We can understand all these observations with the help of the analytical results collected in the previous section. To this end note that, for all the solutions, the brane finds it equilibrium position at a value of $\varphi$ where the bulk potential is well approximated by

\footnotetext{
${ }^{16}$ Here we collect further, but less important observations. For one, we find that there are typically two solutions for the equilibrium position $\varphi_{\star}$ if $\mathcal{R}$ permits a solution. Also, for completeness, note that for $\mathcal{R} \rightarrow 0$ the lower branch in figure 16 (b) is continuously connected to a solution with $\mathcal{R}=0$, whereas this is not the case for the upper branch.
} 
an exponential. ${ }^{17}$ As a result, immediately to the left and the right of the brane the bulk solutions will, at leading order, be given by the solutions collected in section 4.1. More precisely, as the potential is not exactly exponential, the solutions in the full potential will be given by those in section 4.1 up to some small corrections. With this we can explain the results in figure 16 as follows.

- On the IR side of the brane all solutions for $b=1.1 \sqrt{2 / 3}$ are small perturbations of the special solution of type $S=W /(3 b)$ (case 3 ) in the classification of section 4.1. As this is a unique solution only a small subset of solution leaving the UV fixed point will asymptote to this solution. This explains the narrow range in $\mathcal{R}$ for which solutions exist. In addition, for $b=1.1 \sqrt{2 / 3}$ this type of solution has $T \neq 0$, which implies that $\mathcal{R} \neq 0$. Hence we do not expect these solutions to exist for arbitrarily small values of $\mathcal{R}$, which is exactly what we observe. In other words, there is a gap in solutions for $\mathcal{R}>0$.

- In contrast for $b=0.9 \sqrt{2 / 3}$ the solutions on the IR side of the brane are small perturbations of the special solution of type $S=W^{\prime}$ (case 2) in the classification of section 4.1.18 Note that this type of solution has $T=0$ exactly, which would imply $\mathcal{R}=0$. Since the potential is not exactly exponential, the solutions are only approximately of type 2 and finite but small values of $\mathcal{R}$ are allowed. This is exactly what we observe in figure 16 .

Next, we study the brane curvature across our space of solutions. For $b=1.1 \sqrt{2 / 3}$ we plot $R_{B} M_{4}^{-2}$ vs. $\mathcal{R}$ in figure 17 . The main observation is that, unless $\mathcal{R}=0$, the brane curvature $R_{B}$ is always finite and never small. (In fact, for our unrealistic choice of brane parameters it is also extremely super-Planckian.) There is no continuous limit where $R_{B} M_{4}^{-2} \rightarrow 0$.

For $b=0.9 \sqrt{2 / 3}$ the findings are qualitatively different. We display the corresponding results for $R_{B} M_{4}^{-2}$ vs. $\mathcal{R}$ in figure 18. In particular, in figure 18(a) we show results for the lower branch in figure 16(a), while figure 18(b) contains the data for the upper branch of figure 16(a). Most importantly, the lower branch exhibits a limit $R_{B} \rightarrow 0$ for $\mathcal{R} \rightarrow 0$ which is continuously connected to a solution with $R_{B}=0$ and $\mathcal{R}=0$. On the other branch of solutions (figure 18(b)) $R_{B}$ is never zero and potentially diverges for $\mathcal{R} \rightarrow 0 .{ }^{19}$

Last, we comment on the stability of the solutions obtained here. In [20] a set of sufficient criteria (2.56)-(2.59) was derived which guarantee the perturbative stability of

\footnotetext{
${ }^{17}$ Note from figure 16 that for all the solutions obtained the brane equilibrium position takes values $\varphi_{\star} \gtrsim 13$. There the bulk potential is well-approximated by

$$
V=-\frac{V_{1}}{4} e^{b \varphi}\left(1+\mathcal{O}\left(\varphi^{2} e^{-b \varphi}\right)\right)
$$

${ }^{18}$ For $b=0.9 \sqrt{2 / 3}$ solutions of type 3 have $T<0$ and hence $\mathcal{R}<0$. As we restrict our attention to configurations with $\mathcal{R}>0$ we cannot find solutions of type 3 for $b=0.9 \sqrt{2 / 3}$.

${ }^{19}$ We could not determine this decisively in our numerical analysis. While we observe that both $\varphi_{\star}$ and $R_{B} M_{4}^{-2}$ increase on this branch when $\mathcal{R}$ is decreased, we can neither exclude nor confirm whether this continues for arbitrarily small values of $\mathcal{R}$.
} 


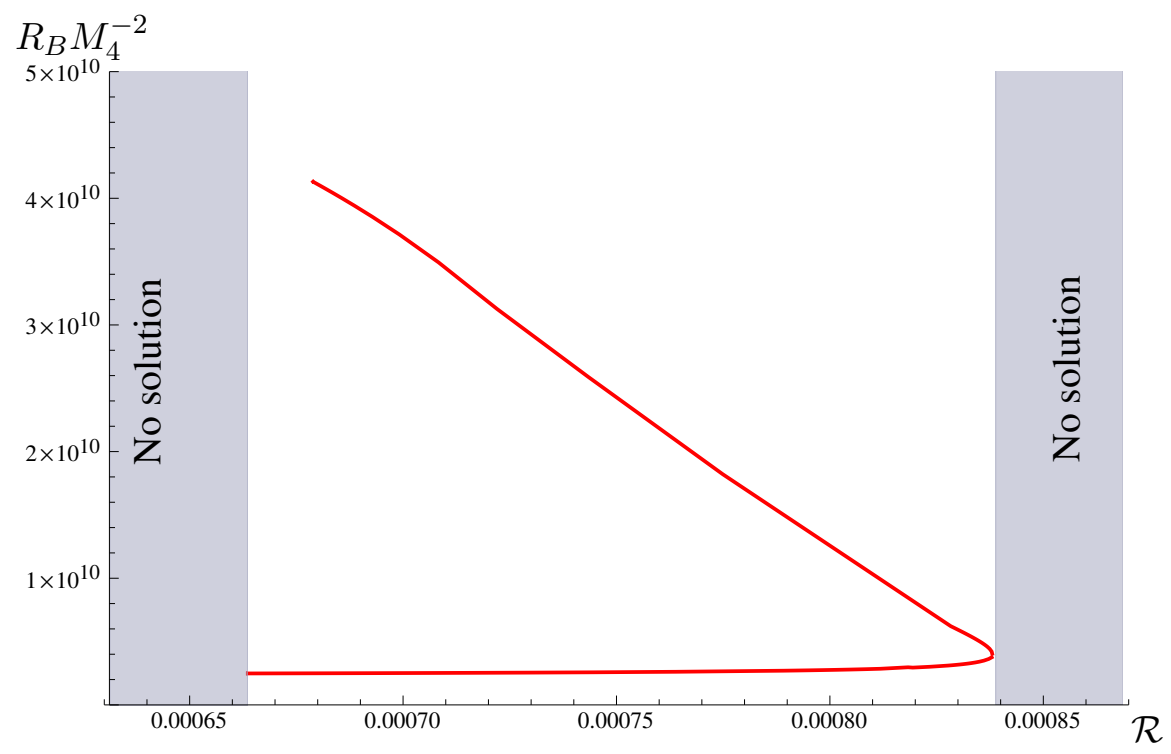

Figure 17. The brane curvature $R_{B} M_{4}^{-2}$ vs. $\mathcal{R}$ for $b=1.1 \times \sqrt{2 / 3}$. Results are obtained for bulk potential (4.1), brane quantities (4.2) and parameter values (4.9).

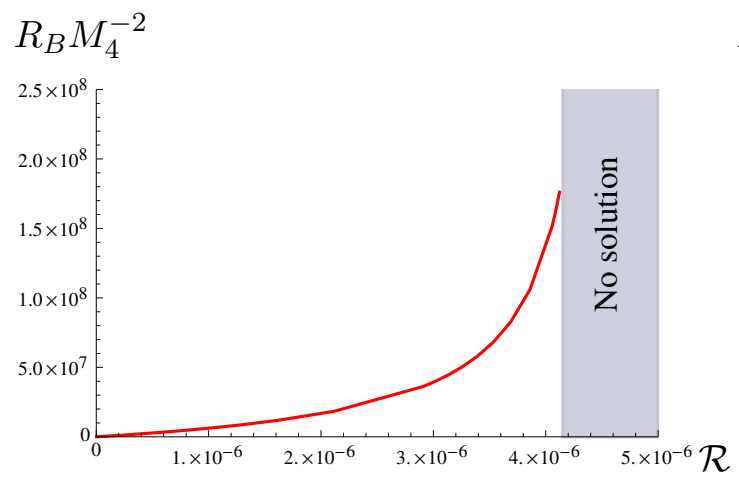

(a)

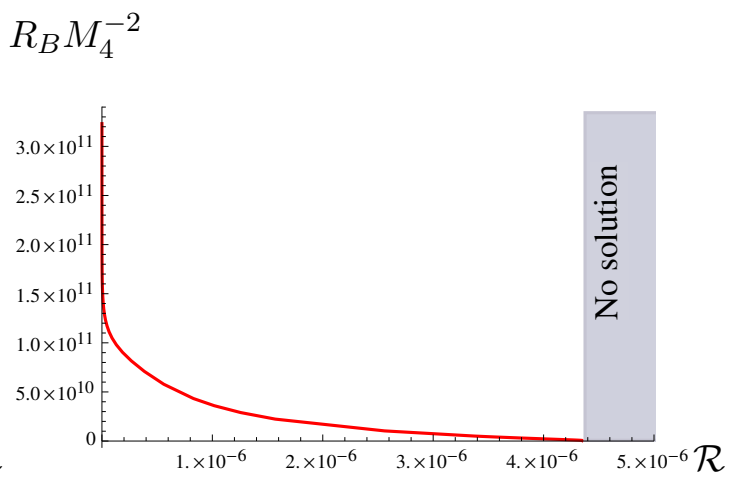

(b)

Figure 18. The brane curvature $R_{B} M_{4}^{-2}$ vs. $\mathcal{R}$ for $b=0.9 \times \sqrt{2 / 3}$. Results are obtained for bulk potential (4.1), brane quantities (4.2) and parameter values (4.9). The plots (a) and (b) exhibit results for the two branches of solutions separately.

a flat brane solution. Here we do find a branch which in the limit $\mathcal{R} \rightarrow 0$ is connected continuously to a flat brane solution. By an explicit calculation we find that this solution satisfies the stability criteria (2.56)-(2.58), but not (2.59). This is not necessarily fatal, as (2.59) is only a sufficient condition for stability. However, a more detailed analysis is necessary to conclusively determine the stability of this solution, which is beyond the scope of this paper. We further expect the stability properties of the flat solution also to extend to the branch of curved brane solutions connected to the flat solution. The reason is that the solutions exhibit positive boundary or brane curvature, which we do not expect to aversely affect stability. 
We are now in a position to summarise our findings for the model studied in this section.

1. We find the space of solutions in $\mathcal{R}$ to be highly restricted. The reason is that for large $\varphi$ for all solutions have to asymptote to one of the two unique solutions. In our case these are the two special solutions for an exponential bulk potential described in section 4.1. Only a small subset of solutions departing from the UV fixed point will asymptote to such a solution and all lie within a narrow range in $\mathcal{R}$.

2. We again find a branch of solutions that in the limit $\mathcal{R} \rightarrow 0$ is connected continuously to a flat brane solution with $\mathcal{R}=0$ and $R_{B}=0$. Here we made sure that flat brane limit satisfies the criteria (2.56)-(2.58) for perturbative stability. It does not satisfy the sufficient condition (2.59).

3. Note that to find solutions satisfying (2.56)-(2.58) in the flat limit we required a bulk potential with infinite range (no minima), a brane potential exhibiting a zero for some value $\varphi>0$ and a sufficiently small value of $U\left(\varphi_{\star}\right)$. No further tuning of parameters beyond this choice is required.

\section{Conclusions and outlook}

In this work we studied self-stabilising solutions of a 4-dimensional brane embedded into a 5-dimensional bulk, where the curvature of the brane is adjusted dynamically. This is in the spirit of self-tuning mechanisms of the cosmological constant in braneworld scenarios [17-20], with the difference that we are not exclusively interested in solutions where the brane is flat.

In particular, the (curved) brane is embedded in a bulk described by Einstein-dilaton gravity with a potential for the dilaton. Following the ideas laid out in [20], our braneworld scenario offers a holographic interpretation. More precisely, the brane-bulk system is dual to a weakly coupled sector (e.g. the Standard Model) interacting with a strongly coupled large $N$ CFT, with the CFT residing on the boundary of the $5 \mathrm{~d}$ bulk, [22]. This is similar to [20], but a new aspect of this analysis is that we also allow the boundary supporting the CFT to be curved. For simplicity, we take the background of the CFT to be a (locally) maximally symmetric 4-dimensional space-time ${ }^{20}\left(\mathrm{dS}_{4}, \mathcal{M}_{4}\right.$ or $\left.\mathrm{AdS}_{4}\right)$ which we characterise by its scalar curvature $R^{(\zeta)}$. The bulk metric can then be written in domain-wall form as

$$
d s^{2}=d u^{2}+e^{2 A(u)} \zeta_{\mu \nu} d x^{\mu} d x^{\nu},
$$

where $\zeta_{\mu \nu}$ is a metric describing the maximally symmetric $4 \mathrm{~d}$ space-time with curvature $R^{(\zeta)}$. Note that $R^{(\zeta)}$ is not dynamical, but fixed as a boundary condition at the conformal boundary of the bulk space-time. The dilaton is also chosen to be constant on the boundary, which in holography corresponds to a constant scalar operator source.

\footnotetext{
${ }^{20}$ As mentioned in previous sections, when the curvature is negative the holographic dictionary is more subtle, and the boundary theory must also includes a defect corresponding to the boundary of the radial slices.
} 
The first observation is that generic self-stabilising solutions with $R^{(\zeta)}=0$ (i.e. $\zeta_{\mu \nu}=$ $\eta_{\mu \nu}$ ) only exist if the world-volume of the brane is also flat. That is, if the boundary CFT resides on Minkowski space, the world-volume of the brane is also given by Minkowski space, which is the scenario studied previously in [20]. Exceptions exist, but are non-generic as they require a tuning of model-parameters (e.g. a precise choice of the dilaton potential on the brane, see appendix A.1 for details). This no-go result can also be overcome if the $5 \mathrm{~d}$ bulk is not static as in (5.1), but time-dependent. However, in this case the dual interpretation in terms of RG flows does not apply any more. In fact, in such a case it corresponds to time dependent dynamics of vevs, associated to non-trivial cosmological evolution on the brane, and we leave this possibility for future work.

To find self-stabilising solutions with a curved brane in a static bulk, one is hence forced to modify the UV boundary conditions of the bulk fields. In this work we mainly did so by choosing $R^{(\zeta)} \neq 0$. We then worked with a simple brane embedding in which the brane geometry is inherited from the boundary. For a bulk described by (5.1) this amounts to locating the brane at some fixed $u=u_{\star}$. This choice is equivalent to restricting to branes with maximally symmetric world-volume with scalar curvature $R_{B}$. The brane curvature is then related to $R^{(\zeta)}$ as

$$
R_{B}=R^{(\zeta)} e^{-2 A\left(u_{\star}\right)}
$$

To find solutions for a brane with world-volume given by $(\mathrm{A}) \mathrm{dS}_{4}$, the boundary CFT has to reside on $(\mathrm{A}) \mathrm{dS}_{4} \cdot{ }^{21}$

There exists an alternative realisation of the same solutions, which can be obtained via a bulk coordinate transformation. In this formulation the boundary metric is flat $\left(R^{(\zeta)}=0\right)$, but the scalar sources are no longer constant on the boundary: they now vary in space or time. As a result, the holographic interpretation is also modified. Instead of the boundary QFT living on a curved space-time, we then have a flat-space QFT driven by a time-varying (in the $\mathrm{dS}$ case) or space-dependent (in the AdS case) source. In the $\mathrm{dS}$ case one requires the source $j$ to vary as $j \sim|t|^{\Delta-d}$, where $t$ is the de Sitter conformal time on the brane and $\Delta$ is the dimension of the relevant operator deforming the CFT.

We then studied how the brane curvature $R_{B}$ depends on $R^{(\zeta)}$ quantitatively. This was done mostly numerically by searching for self-stabilising solutions while scanning over all possible values of $R^{(\zeta)}$. To perform a numerical analysis, we have to specify a particular model by choosing a bulk dilaton potential $V(\varphi)$, a brane dilaton potential $W_{B}(\varphi)$, and the (dilaton-dependent) Newton's 'constant' term $U(\varphi)$ on the brane. Given a UV completion of our model, these functions can in principle be determined, but this goes beyond the scope of this work. Here we chose generic functions for $V(\varphi), W_{B}(\varphi)$ and $U(\varphi)$ and studied the consequences for self-stabilisation. To assess how model-dependent our findings are, we performed our analysis for three different combinations of $V(\varphi), W_{B}(\varphi)$ and $U(\varphi)$. To be specific, we compared setups with restricted vs. unlimited dilaton range. We also contrasted the effect of choosing $U(\varphi)$ to be fast-varying vs. constant in $\varphi$. Rather than trying to

\footnotetext{
${ }^{21}$ As shown in [33] such AdS-sliced bulk solutions have twin boundary singularities that also affect the brane. They could be resolved by lower codimension branes.
} 
construct a phenomenologically viable model, we were more interested in exploring the scope of effects that can arise in this brane-bulk system.

While the precise numerical results are model-dependent, we observed similar qualitative features in all three examples studied:

- For any given value of $R^{(\zeta)}$ there typically exists at least one self-stabilising solution. Frequently, there exist several branches of solutions as a function of $R^{(\zeta)}$, which differ in the values for the brane position $\varphi_{\star}=\varphi\left(u_{\star}\right)$ and the brane curvature $R_{B}$.

- For $R^{(\zeta)} \rightarrow 0$ one of the branches connects continuously to a solution with $R^{(\zeta)}=0$ and $R_{B}=0$, i.e. a flat brane as studied in [20]. Hence there exists a limit where $R_{B}$ can be made arbitrarily small by letting $R^{(\zeta)} \rightarrow 0$ in a controlled way. Thus we can obtain solutions with parametrically small $R_{B}$ in this setup.

- Interestingly, there also exist solutions where $R_{B}$ stays finite for $R^{(\zeta)} \rightarrow 0$. In this case there is no solution with $R^{(\zeta)}=0$ exactly. The reason is that the "would-be solution' with $R^{(\zeta)}=0$ can be shown to be a solution with finite $R^{(\zeta)}$ associated with a different UV fixed point. This effect is also observed for holographic RG flows in absence of a brane $[33,56]$.

- Last, for solutions with $R^{(\zeta)} \rightarrow \infty$, the brane curvature does not diverge, but rather asymptotes to a finite value.

- However, for the models studied here, the typical scale of the brane curvature is the 4d Planck scale $M_{4}$ on the brane, i.e. $R_{B} \sim M_{4}^{2}$. Only on the branch connected to the flat brane solution we can achieve $R_{B} \ll M_{4}^{2}$ by tuning $R^{(\zeta)}$ to a sufficiently small value.

These findings make the bulk-brane setup studied here a promising framework for further phenomenological investigation. However, before a realistic model can be constructed, there are several open questions that should be addressed. For one, it is important to determine to what extent our results above are general, or model-dependent artifacts. In particular, is it a generic feature of this construction that $R_{B} \ll M_{4}^{2}$ only occurs when perturbing a solution for a flat brane?

In addition, more work is also needed regarding the theoretical foundations of the model. It would be desirable if the quantities $V(\varphi), W_{B}(\varphi)$ and $U(\varphi)$ could be constrained, either by direct calculation or from physical principles. For example, it is expected that consistency with quantum gravity gives stringent constraints on the physics of scalar fields [46, 57-62], restricting their field range and even constraining the shape of the potential. It would be interesting to study to what extent these conditions, also known as 'swampland conjectures', can be used to constrain this model.

Another important question regards the stability of the solutions obtained here. The perturbative stability of self-stabilising solutions for a flat brane was analysed in [20]. The result of this analysis is a set of five sufficient conditions for stability involving the bulk solutions and the brane quantities evaluated at the position of the brane. Here, we expect 
that the presence of positive brane curvature will not introduce any additional instabilities (see [33] for more details). Hence we expect any solutions, which can be obtained from a stable flat brane solution by turning on positive (boundary and brane) curvature to be stable. On the other hand, the presence of negative (boundary and brane) curvature may introduce new instabilities. In this case, perturbative stability has to be checked explicitly case by case, which goes beyond the scope of this work. As our priority in this work was to explore the space of self-stabilising solutions rather than to perform realistic modelbuilding, the solutions explored in this work do not always satisfy all of the sufficient conditions for stability. For example, the solutions in section 4 satisfy all but one of the sufficient stability conditions. This does not imply that these solutions are necessarily unstable, but a more detailed analysis is required.

Last, some of our findings may have interesting applications in the study of holographic RG flows. In particular, certain bulk solutions studied in the theory with $V(\varphi) \sim-\exp (\varphi)$ should be relevant for the study of the RG behaviour of confining theories [27] on curved backgrounds in holography. We plan to report on this in a separate work in the future.

\section{Acknowledgments}

We thank Christos Charmousis, Jerome Gauntlett, Dieter Lüst, Hiroshi Ooguri, Ioannis Papadimitriou, Cumrun Vafa for discussions and comments.

This work was supported in part by the Advanced ERC grant SM-grav, No 669288.

\section{A Junction conditions for curved brane embeddings in a flat-sliced bulk}

Here we consider a brane describing a curve $u=u_{\star}(\tau)$, which constitutes the interface between solutions of the form

$$
d s^{2}=d u^{2}+e^{2 A(u)} \eta_{\mu \nu} d x^{\mu} d x^{\nu}, \quad \varphi=\varphi(u)
$$

in which the scale factor and scalar field profile are, a priori, different on each side of the interface,

$$
(A, \varphi)= \begin{cases}\left(A_{-}(u), \varphi_{-}(u)\right) & u<u_{\star}(\tau) \\ \left(A_{+}(u), \varphi_{+}(u)\right) & u>u_{\star}(\tau)\end{cases}
$$

The connection across the brane is specified by Israel's junction conditions:

1. The metric and scalar field are continuous:

$$
\left[g_{a b}\right]_{\mathrm{IR}}^{\mathrm{UV}}=0, \quad[\varphi]_{\mathrm{UV}}^{\mathrm{IR}}=0
$$

2. The extrinsic curvature and normal derivative of $\varphi$ are discontinuous:

$$
\left[K_{\mu \nu}-\gamma_{\mu \nu} K\right]_{\mathrm{UV}}^{\mathrm{IR}}=\frac{1}{\sqrt{-\gamma}} \frac{\delta S_{\text {brane }}}{\delta \gamma^{\mu \nu}}, \quad\left[n^{a} \partial_{a} \varphi\right]_{\mathrm{UV}}^{\mathrm{IR}}=-\frac{1}{\sqrt{-\gamma}} \frac{\delta S_{\text {brane }}}{\delta \varphi},
$$

where $\gamma_{\mu \nu}=e^{2 A(u)} \zeta_{\mu \nu}$ is the induced metric, $K_{\mu \nu}$ is the extrinsic curvature of the brane with trace $K=\gamma^{\mu \nu} K_{\mu \nu}$, and $n^{a}$ a unit vector normal to the brane with orientation towards the IR. 
The first of these conditions, the continuity of the metric and scalar field across the interface, implies

$$
A_{-}\left(u_{\star}(\tau)\right)=A_{+}\left(u_{\star}(\tau)\right), \quad \varphi_{-}\left(u_{\star}(\tau)\right)=\varphi_{+}\left(u_{\star}(\tau)\right) .
$$

If $u_{\star}(\tau)$ is a non-trivial function, equation (A.5) implies the identity of the functions $A_{-}(u)$ and $A_{+}(u)$, and of $\varphi_{-}(u)$ and $\varphi_{+}(u)$, over a continuous set of values. Since in the bulk these functions satisfy a system of ordinary differential equations, this implies that the solutions on each side must coincide,

$$
A_{-}(u)=A_{+}(u), \quad \varphi_{-}(u)=\varphi_{+}(u), \quad \forall u .
$$

Therefore, not only $A$ and $\varphi$ but also their derivatives must be continuous. Then, the second junction conditions require

$$
\frac{\delta S_{\text {brane }}}{\delta \gamma^{\mu \nu}}=0, \quad \frac{\delta S_{\text {brane }}}{\delta \varphi}=0 .
$$

In other words, the induced metric and the scalar on the brane must satisfy their lowerdimensional field equations, as dictated by the brane action alone. Recall however that the induced metric $\gamma_{\mu \nu}$ and the brane scalar field $\varphi$ are not independent quantities, but they are determined by the bulk metric and scalar field, via the embedding function $u_{\star}(\tau)$ : therefore, generically the solution of equations (A.7) will be incompatible with the bulk solution.

To illustrate this more explicitly, we write the induced metric and scalar field for a general embedding $u_{*}(t)$ :

$$
d s_{\text {ind }}^{2}=\left[\left(\frac{d u_{\star}}{d \tau}\right)^{2}-e^{2 A\left(u_{\star}(\tau)\right)}\right] d \tau^{2}+e^{2 A\left(u_{\star}(\tau)\right)} d x_{i} d x^{i}, \quad \phi(\tau)=\varphi\left(u_{\star}(\tau)\right)
$$

where we have used a different notation $\phi(\tau)$ to denote the induced scalar field. We can change coordinates on the brane to proper time $\eta$, where the induced metric takes the canonical FRW form

$$
d s_{i n d}^{2}=-d \eta^{2}+a^{2}(\eta) d x_{i} d x^{i}, \quad a(\eta) \equiv e^{A\left(u_{\star}(\eta)\right)} .
$$

Because of (A.7), the induced scale factor $a(\eta)$ and scalar field $\phi(\eta)$ must satisfy the brane Einstein-scalar equations, whose solution is determined purely by the brane potentials without reference to the bulk.

Given a solution $(a(\eta), \phi(\eta))$ of the brane Einstein's equations and knowing the bulk geometry $A(u)$ we can determine the embedding $u_{\star}(\eta)$ by inverting the implicit relation

$$
A\left(u_{\star}(\eta)\right)=\log a(\eta)
$$

Having found $u_{\star}(\eta)$ we can go back to the bulk time coordinate $\tau$ by solving the differential equation

$$
\frac{d \eta}{d \tau}=\frac{a(\eta)}{\left[1+\left(\frac{d u_{\star}}{d \eta}\right)^{2}\right]^{1 / 2}}
$$

which follows from the change of coordinates between (A.8) and (A.9). 
The embedding $u_{\star}(\eta)$ must be such that, at the same time as (A.10), one must also satisfy the relation

$$
\varphi\left(u_{\star}(\eta)\right)=\phi(\eta)
$$

On the other hand, the functions $A(u)$ and $\varphi(u)$ are determined by the bulk Einstein equations, which generically know nothing about the brane potentials. Therefore, if we determine $u_{\star}(\tau)$ from knowledge of $a(\eta)$ and $A(u)$ as explained above, generically the relation (A.12) will not hold, and we are forced to conclude that the ansatz we started from does not lead to a solution of the full system.

The argument above assumes generic (and unrelated) bulk and brane potentials. However, if we abandon genericity, it may be possible to tune the model such that equations (A.10)-(A.12) are indeed compatible, and a solution exists. This leads to the curious case which we call an evanescent brane, i.e. an exact solution of the bulk-brane system in which the brane has no backreaction on the bulk.

\section{A.1 Evanescent branes}

As we have seen in the previous discussion, embedding a non-trivial brane trajectory in a flat-slicing is possible if the induced quantities on the brane satisfy their lower-dimensional field equations governed by the brane potentials. If that is the case, the bulk is smooth across the brane, and the interface is transparent (or invisible), although all bulk equations and junction conditions are exactly satisfied: curiously, we have a fully backreacted system where the backreaction is exactly vanishing.

A simple example of such a situation is given by a bulk solution which is Poincaré-AdS with constant scalar field (realised e.g. at an extremum of $V(\varphi)$, say at $\varphi=0$ ),

$$
A(u)=-\frac{u}{\ell}, \quad \varphi(u)=0,
$$

and a brane action of the form (2.3) with constant $U$ and $Z$ and a potential $W_{B}(\varphi)$ such that it also has an extremum at $\varphi=0$, with $W_{B}(0)>0$. In this case, the brane field equations (A.7) admit a de Sitter solution with constant scalar $\phi=0$ and Hubble constant $H=\sqrt{W_{B}(0)} / M_{p}^{2}$, where $M_{p}^{2}=M^{3} U$,

$$
a(\eta)=e^{H \eta}, \quad \phi(\eta)=0 .
$$

Comparing equations (A.13) and (A.14) we can read-off the trajectory using equation (A.10),

$$
u_{\star}(\eta)=-\ell H \eta
$$

Equation (A.11) becomes

$$
\frac{d \eta}{d \tau}=\frac{e^{H \eta}}{\left[1+H^{2} \ell^{2}\right]^{1 / 2}},
$$

and by integrating it we can find the trajectory in the original bulk coordinates,

$$
u_{\star}(\tau)=\ell \log \left[-\frac{H}{\left(1+H^{2} \ell^{2}\right)^{1 / 2}} \tau\right], \quad-\infty<\tau<0 .
$$


From the brane point of view, $\tau$ is the de Sitter conformal time. Finally, and crucially, $\varphi(u(\tau))=\phi(\tau)$ since both sides vanish identically, by equations (A.13)-(A.14). Therefore, we have an exact solution of the full system, including the junction conditions. This was possible because we have tuned the brane theory such that an extremum of the brane potential coincides with an extremum of the bulk potential. It is likely that similar examples can be constructed with a non-trivial bulk scalar field profile, e.g. by appropriate combinations of bulk and brane exponential potentials.

We stress that in these solutions the bulk does not detect at all the presence of the brane: the bulk AdS solution would be the same were the brane absent. What we have here is a non-trivial generalization of the fact that, if the world-volume action has only a potential term, then a tensionless brane produces no backreaction. In our case instead, we have a non-vanishing tension, but induced kinetic terms for gravity and the scalar. The corresponding statement is that a brane satisfying its own world-volume Einstein equation behaves (from the point of view of the bulk) as if it were tensionless.

\section{B Perturbative analysis near the maximum of the potential}

Here we record expressions for the functions $W, S$ and $T$ defined in (2.21)-(2.23) in the vicinity of a maximum of the potential. Without a loss of generality, we can consider the maximum to be located at $\varphi=0$ and near this maximum the potential can be written as

$$
V(\varphi)=-\frac{d(d-1)}{\ell^{2}}+\frac{m^{2}}{2} \varphi^{2}+\mathcal{O}\left(\varphi^{3}\right)
$$

where $m^{2}<0$. We can now solve eqs. (2.28)-(2.29) in a series expansion in $\varphi$. There exist two types of solutions which are distinguished by the subscripts $(+)$ and $(-)$, respectively. The (-) solutions are:

$$
\begin{aligned}
W_{-}(\varphi)= & \frac{1}{\ell}\left[2(d-1)+\frac{\Delta_{-}}{2} \varphi^{2}+\mathcal{O}\left(\varphi^{3}\right)\right]+\frac{\mathcal{R}}{d \ell}|\varphi|^{\frac{2}{\Delta_{-}}}[1+\mathcal{O}(\varphi)+\mathcal{O}(\mathcal{R})] \\
& +\frac{C}{\ell}|\varphi|^{\frac{d}{\Delta_{-}}}[1+\mathcal{O}(\varphi)+\mathcal{O}(C)+\mathcal{O}(\mathcal{R})], \\
S_{-}(\varphi)= & \frac{\Delta_{-}}{\ell} \varphi[1+\mathcal{O}(\varphi)]+\frac{C d}{\Delta_{-} \ell}|\varphi|^{\frac{d}{\Delta_{-}}-1}[1+\mathcal{O}(\varphi)+\mathcal{O}(C)], \\
& +\frac{1}{\ell} \mathcal{O}\left(\mathcal{R}|\varphi|^{\frac{2}{\Delta_{-}}}+1\right)+\frac{1}{\ell} \mathcal{O}\left(\mathcal{R} C|\varphi|^{\frac{2+d}{\Delta_{-}}-1}\right) \\
T_{-}(\varphi)= & \ell^{-2} \mathcal{R}|\varphi|^{\frac{2}{\Delta_{-}}}[1+\mathcal{O}(\varphi)+\mathcal{O}(C)+\mathcal{O}(\mathcal{R})],
\end{aligned}
$$

where $C$ and $\mathcal{R}$ are two integration constants and

$$
\Delta_{ \pm}=\frac{1}{2}\left(d \pm \sqrt{d^{2}+4 m^{2} \ell^{2}}\right) \quad \text { with } \quad-\frac{d^{2}}{4 \ell^{2}}<m^{2}<0 .
$$


The mass bound is precisely the $\mathrm{BF}$ bound for a scalar in $d$ dimensions. On the other hand, the $(+)$ solutions are:

$$
\begin{aligned}
W_{+}(\varphi) & =\frac{1}{\ell}\left[2(d-1)+\frac{\Delta_{+}}{2} \varphi^{2}+\mathcal{O}\left(\varphi^{3}\right)\right]+\frac{\mathcal{R}}{d \ell}|\varphi|^{\frac{2}{\Delta_{+}}}[1+\mathcal{O}(\varphi)+\mathcal{O}(\mathcal{R})], \\
S_{+}(\varphi) & =\frac{\Delta_{+}}{\ell} \varphi[1+\mathcal{O}(\varphi)]+\mathcal{O}\left(\mathcal{R}\left|\varphi_{-}\right|^{\frac{2}{\Delta_{+}}}+1\right. \\
T_{+}(\varphi) & =\ell^{-2} \mathcal{R}|\varphi|^{\frac{2}{\Delta_{+}}}[1+\mathcal{O}(\varphi)+\mathcal{O}(\mathcal{R})] .
\end{aligned}
$$

For the (-) solutions both integration constants $\mathcal{R}$ and $C$ appear, but for the $(+)$ solutions $\mathcal{R}$ is the only integration constant.

The solutions $(W, S, T)$ consist of two parts. One is an analytic series in $\varphi$ and the other consists of non-analytic expansions in $\varphi$ also containing the integration constants. In fact we can write the $W_{-}$solution as a triple expansion as

$$
W_{-}(\varphi)=\frac{1}{\ell} \sum_{l=0}^{\infty} \sum_{m=0}^{\infty} \sum_{n=0}^{\infty} A_{l, m, n}\left(C|\varphi|^{d / \Delta_{-}}\right)^{l}\left(\mathcal{R}|\varphi|^{2 / \Delta_{-}}\right)^{m} \varphi^{n} .
$$

On the other hand, the $W_{+}$solution can be written as a double expansion, which is schematically given by

$$
W_{+}(\varphi)=\frac{1}{\ell} \sum_{m=0}^{\infty} \sum_{n=0}^{\infty} A_{m, n}\left(\mathcal{R}|\varphi|^{2 / \Delta_{-}}\right)^{m} \varphi^{n} .
$$

Given the expressions of the functions $W(\varphi)$ and $S(\varphi)$, we can use the definitions (2.21) and (2.22) to find the scale factor $A(u)$ and the scalar field profile $\varphi(u)$. These are recorded below. For the $(-)$ solutions they are:

$$
\begin{aligned}
\varphi(u)= & \varphi_{-} \ell^{\Delta_{-}} e^{\Delta_{-} u / \ell}\left[1+\mathcal{O}\left(\mathcal{R}\left|\varphi_{-}\right|^{2 / \Delta_{-}} e^{2 u / \ell}\right)+\ldots\right] \\
& +\frac{C d\left|\varphi_{-}\right|^{\Delta_{+} / \Delta_{-}}}{\Delta_{-}\left(d-2 \Delta_{-}\right)} \ell^{\Delta_{+}} e^{\Delta_{+} u \ell}\left[1+\mathcal{O}\left(\mathcal{R}\left|\varphi_{-}\right|^{2 / \Delta_{-}} e^{2 u / \ell}\right)+\ldots\right]+\ldots, \\
A(u)= & \bar{A}_{-}-\frac{u}{\ell}-\frac{\varphi_{-}^{2} \ell^{2 \Delta_{-}}}{8(d-1)} e^{2 \Delta_{-} u / \ell}-\frac{\mathcal{R}\left|\varphi_{-}\right|^{2 / \Delta_{-}} \ell^{2}}{4 d(d-1)} e^{2 u / \ell} \\
& -\frac{\Delta_{+} C\left|\varphi_{-}\right|^{d / \Delta_{-}} \ell^{d}}{d(d-1)\left(d-2 \Delta_{-}\right)} e^{d u / \ell}+\ldots
\end{aligned}
$$

where $\varphi_{-}$and $\bar{A}_{-}$are integration constants. For the $(+)$solutions one obtains:

$$
\begin{aligned}
& \varphi(u)=\varphi_{+} \ell^{\Delta_{+}} e^{\Delta_{+} u / \ell}\left[1+\mathcal{O}\left(\mathcal{R}\left|\varphi_{+}\right|^{2 / \Delta_{+}} e^{2 u / \ell}\right)+\ldots\right]+\ldots, \\
& A(u)=\bar{A}_{+}-\frac{u}{\ell}-\frac{\varphi_{+}^{2} \ell^{2 \Delta_{+}}}{8(d-1)} e^{2 \Delta_{+} u / \ell}-\frac{\mathcal{R}\left|\varphi_{+}\right|^{2 / \Delta_{+}} \ell^{2}}{4 d(d-1)} e^{2 u / \ell}+\ldots,
\end{aligned}
$$

with integration constants $\varphi_{+}$and $\bar{A}_{+}$.

As we are solving the equations of motion close to $\varphi=0$, from eqs. (B.11) and (B.13) we can see that these solutions are valid near $u \rightarrow-\infty$. Choosing $A_{ \pm}=0$, which can be 
done by a redefinition of the boundary coordinates, is equivalent to the choice $\zeta_{\mu \nu}=\zeta_{\mu \nu}^{\mathrm{UV}}$ made in (2.6).

The integration constant $\varphi_{-}$is interpreted as the source for the operator $\mathcal{O}$ of the boundary QFT. The dimension of this operator is $\Delta_{+}$and it is related to the mass parameter $m$ by eq. (B.5). The integration constant $C$ is related to the vacuum expectation value of the scalar operator and it is given by

$$
\langle\mathcal{O}\rangle=\frac{C d}{\Delta_{-}}\left|\varphi_{-}\right|^{\Delta_{+} / \Delta_{-}} .
$$

For the $(+)$ solution, the source vanishes. The flow corresponding to the $(+)$ solution is thus driven purely by the vev. In this case the vev of the operator $\mathcal{O}$ is related to the integration constant $\varphi_{+}$by

$$
\langle\mathcal{O}\rangle_{+}=\left(2 \Delta_{+}-d\right) \varphi_{+}
$$

The integration constant $\mathcal{R}$ is dimensionless. This is the dimensionless combination of the parameters of the theory namely source $\varphi_{-}$and the boundary curvature $R^{\mathrm{UV}}$. More precisely the relation is

$$
\mathcal{R}=R^{(\zeta)}\left|\varphi_{\mp}\right|^{-2 / \Delta_{\mp}} .
$$

To conclude this section, we see that the integration constants are related to the different parameters of the boundary field theory. We also see that the bulk geometry asymptotes to $\mathrm{AdS}_{d+1}$ near the maximum of the potential. From the boundary QFT point of view, this corresponds to the UV fixed point of the RG flows.

\section{Regular IR geometries}

After analyzing the solution near the maximum of the potential which corresponds to the UV fixed point of the RG flows of the boundary field theory, we now analyze the regular solutions in the interior. We are interested in how the scale factor $A(u)$ can obtain its minimum value. For the positively curved case, this will correspond to the regular IR end points. On the other hand, for the negatively curved case, this will correspond to a turning point. In the course of obtaining the solution, a third possibility also arises which we call a bounce and where the flow reverses direction in $\varphi$.

We want to analyze the solution near $\varphi=\varphi_{0}$ where $S\left(\varphi_{0}\right)=0$. As explained in [33], in the vicinity of $\varphi_{0}$ we can expand the functions $W, S$ and $T$ in powers of the square root of $x=\varphi_{0}-\varphi$ :

$$
\begin{aligned}
S(x) & =\sqrt{x}\left(S_{0}+S_{1} \sqrt{x}+\cdots\right), \\
W(x) & =\frac{1}{\sqrt{x}}\left(W_{0}+W_{1} \sqrt{x}+\cdots\right), \\
T(x) & =\frac{1}{x}\left(T_{0}+T_{1} \sqrt{x}+\cdots\right) .
\end{aligned}
$$

As $\varphi_{0}$ is a generic point the potential $V(\varphi)$ has a regular series expansion:

$$
V(\varphi)=V_{0}+V_{1} x+V_{2} x^{2}+\cdots
$$


The unknown coefficients can be determined by plugging expansions (C.1) and (C.2) into eqs. (2.25), (2.28) and (2.29). We find three classes of solutions.

Case (a): IR endpoints. In this case the leading order terms in the functions $S(\varphi), W(\varphi)$ and $T(\varphi)$ are:

$$
\begin{aligned}
S(\varphi) & \approx S_{0} \sqrt{\varphi_{0}-\varphi} \\
S(\varphi) & \approx \frac{W_{0}}{\sqrt{\varphi_{0}-\varphi}}, \\
T(\varphi) & \approx \frac{W_{0}}{\varphi_{0}-\varphi} .
\end{aligned}
$$

where the coefficients are given by:

$$
S_{0}^{2}=\frac{2 V_{1}}{d+1}, \quad W_{0}=(d-1) S_{0}, \quad T_{0}=\frac{d(d-1)}{4(d+1)} S_{0}^{2} .
$$

Note that the function $T(\varphi)$ diverges when $\varphi \rightarrow \varphi_{0}$. From the definition of $T=R^{(\zeta)} e^{-2 A(u)}$, it means that the scale factor, $A(u)$, is shrinking to zero. As the value of $T_{0}$ is positive for $d>1$, this case corresponds to the IR end point of the corresponding RG flow in positively curved space.

Case (b): AdS throat. In this case, the leading order terms of the functions $S, W$ and $T$ are:

$$
\begin{aligned}
S(\varphi) & \simeq S_{0} \sqrt{\varphi_{0}-\varphi}+\cdots \\
W(\varphi) & \simeq W_{0} \sqrt{\varphi_{0}-\varphi}+\cdots \\
T(\varphi) & \simeq T_{2}+T_{3} \sqrt{\varphi_{0}-\varphi}+\cdots,
\end{aligned}
$$

where

$$
S_{0}^{2}=2 V_{1}, \quad W_{2}=\frac{4 V_{0}}{d S_{0}}, \quad T_{2}=V_{0}
$$

In this case, both the functions $S(\varphi)$ and $W(\varphi)$ are going to zero as $\varphi \rightarrow \varphi_{0}$. This means that both the scalar field and the scale factor reach an extremum. As $\ddot{A}=W^{\prime} S>0$ when $\varphi \rightarrow \varphi_{0}$, the scale factor is attaining its minimum value at this point. On the other hand, the function $T(\varphi)$ obtains a constant negative value. Hence, this case corresponds to the turning point of the scale factor for the negatively curved space.

Case (c): bouncing points. In the third case the leading order terms of the functions $S, W$ and $T$ are:

$$
\begin{aligned}
S(\varphi) & \simeq S_{0} \sqrt{\varphi_{0}-\varphi}+\cdots, \\
W(\varphi) & \simeq W_{1}+W_{2} \sqrt{\varphi_{0}-\varphi}+\cdots, \\
T(\varphi) & \simeq T_{2}+T_{3} \sqrt{\varphi_{0}-\varphi}+\cdots,
\end{aligned}
$$


where

$$
S_{0}^{2}=2 V_{1}, \quad T_{2}=V_{0}+\frac{d W_{1}^{2}}{4(d-1)},
$$

and in this case $W_{1}$ is arbitrary. The function $S(\varphi)$ goes to zero when $\varphi \rightarrow \varphi_{0}$. This means that the scalar field is obtaining its extremum value. As $\ddot{\varphi}=S S^{\prime} \sim V_{1}$, the scalar field can obtain its maximum or minimum value depending on the sign of $V_{1}$. On the other hand the function $W(\varphi)$ obtains a finite value $W_{1}$ when $\varphi \rightarrow \varphi_{0}$ which indicates that the scale factor is not attaining its minimum value at this point. This means that the scalar field changes its direction and continues to flow. This refers to the bouncing point and the bouncing flows are expected for both the positively and the negatively curved space.

\section{Perturbative solution of the junction conditions near a flat equilibrium point}

We assume that the equilibrium brane position $\varphi_{\star}$ has the following curvature expansion:

$$
\varphi_{\star}=\varphi_{\star, 0}+R^{(\zeta)} \varphi_{\star, 1}+\cdots
$$

where $\varphi_{\star, 0}$ is the flat brane position and $R^{(\zeta)} \varphi_{\star, 1}$ is the leading-order curvature correction. To remove clutter, we will drop the superscript on $R^{(\zeta)}$ in the following and simply write $R$. Then we can solve eq. (2.54) order by order in $R$. The functions $W, S$ and $T$ have the following curvature expansion:

$$
\begin{aligned}
W(\varphi) & =W_{0}(\varphi)+R W_{1}(\varphi)+\cdots \\
S(\varphi) & =S_{0}(\varphi)+R S_{1}(\varphi)+\cdots \\
T(\varphi) & =R T_{0}(\varphi)+\cdots
\end{aligned}
$$

Zeroth order

The junction condition at zeroth order in $R$ is given by

$$
\left[-2 Q^{2} W_{0} W_{B}+Q^{2} W_{B}^{2}+2 W_{0}^{\prime} W_{B}^{\prime}-\left(W_{B}^{\prime}\right)^{2}\right]_{\varphi_{\star}, 0}=0,
$$

where we have used $S_{0}=W_{0}^{\prime}$. Note that the function $U(\varphi)$ does not appear in the zeroth order equation. This is because in eq. (2.54) the term with the function $U(\varphi)$ is first order in $R$. The solution to eq. (D.5) will determine the brane position in the case of flat slicing.

\section{Linear order}

Equating the coefficients of $R$ in eq. (2.54) we find

$$
\begin{aligned}
& {\left[Q^{2} W_{1} W_{B}+Q^{2} \varphi_{\star, 1} W_{B} W_{0}^{\prime}+T_{0}\left\{\frac{2-d}{d} Q^{2} U\left(W_{0}-W_{B}\right)+U^{\prime}\left(S_{0}-W_{B}^{\prime}\right)\right\}\right.} \\
& -S_{1} W_{B}^{\prime}+Q^{2} \varphi_{\star, 1} W_{0} W_{B}^{\prime}-Q^{2} \varphi_{\star, 1} W_{B} W_{B}^{\prime}-\varphi_{\star, 1} S_{0}^{\prime} W_{B}^{\prime}-\varphi_{\star, 1} S_{0} W_{B}^{\prime \prime} \\
& \left.+\varphi_{\star, 1} W_{B}^{\prime} W_{B}^{\prime \prime}\right]_{\varphi_{\star, 0}}=0 .
\end{aligned}
$$


This equation is linear in $\varphi_{\star, 1}$ and can be easily inverted to get an expression for $\varphi_{\star, 1}$. This is recorded below

$$
\varphi_{\star, 1}=\left.\frac{\frac{(2-d) Q^{2}}{d}\left(T_{0} U W_{0}-T_{0} U W_{B}\right)+Q^{2} W_{1} W_{B}+S_{0} T_{0} U^{\prime}-S_{1} W_{B}^{\prime}-T_{0} U^{\prime} W_{B}^{\prime}}{-Q^{2}\left(W_{B} W_{0}^{\prime}+W_{0} W_{B}^{\prime}-W_{B} W_{B}^{\prime}\right)+S_{0}^{\prime} W_{B}^{\prime}+S_{0} W_{B}^{\prime \prime}-W_{B}^{\prime} W_{B}^{\prime \prime}}\right|_{\varphi_{\star}, 0} .
$$

This is the first leading curvature correction to the equilibrium brane position $\varphi_{\star}$.

Open Access. This article is distributed under the terms of the Creative Commons Attribution License (CC-BY 4.0), which permits any use, distribution and reproduction in any medium, provided the original author(s) and source are credited.

\section{References}

[1] J.M. Maldacena, The Large $N$ limit of superconformal field theories and supergravity, Int. J. Theor. Phys. 38 (1999) 1113 [hep-th/9711200] [INSPIRE].

[2] E. Witten, Anti-de Sitter space and holography, Adv. Theor. Math. Phys. 2 (1998) 253 [hep-th/9802150] [INSPIRE].

[3] S.S. Gubser, I.R. Klebanov and A.M. Polyakov, Gauge theory correlators from noncritical string theory, Phys. Lett. B 428 (1998) 105 [hep-th/9802109] [INSPIRE].

[4] I. Antoniadis, N. Arkani-Hamed, S. Dimopoulos and G.R. Dvali, New dimensions at a millimeter to a Fermi and superstrings at a TeV, Phys. Lett. B 436 (1998) 257 [hep-ph/9804398] [INSPIRE].

[5] L. Randall and R. Sundrum, A Large mass hierarchy from a small extra dimension, Phys. Rev. Lett. 83 (1999) 3370 [hep-ph/9905221] [INSPIRE].

[6] L. Randall and R. Sundrum, An Alternative to compactification, Phys. Rev. Lett. 83 (1999) 4690 [hep-th/9906064] [INSPIRE].

[7] J. Maldacena, unpublished.

[8] S.S. Gubser, AdS/CFT and gravity, Phys. Rev. D 63 (2001) 084017 [hep-th/9912001] [INSPIRE].

[9] N. Arkani-Hamed, M. Porrati and L. Randall, Holography and phenomenology, JHEP 08 (2001) 017 [hep-th/0012148] [INSPIRE].

[10] P. Binetruy, C. Deffayet and D. Langlois, Nonconventional cosmology from a brane universe, Nucl. Phys. B 565 (2000) 269 [hep-th/9905012] [INSPIRE].

[11] J.M. Cline, C. Grojean and G. Servant, Cosmological expansion in the presence of extra dimensions, Phys. Rev. Lett. 83 (1999) 4245 [hep-ph/9906523] [INSPIRE].

[12] P. Binetruy, C. Deffayet, U. Ellwanger and D. Langlois, Brane cosmological evolution in a bulk with cosmological constant, Phys. Lett. B 477 (2000) 285 [hep-th/9910219] [InSPIRE].

[13] C. Deffayet, Cosmology on a brane in Minkowski bulk, Phys. Lett. B 502 (2001) 199 [hep-th/0010186] [INSPIRE].

[14] C. Deffayet, G.R. Dvali and G. Gabadadze, Accelerated universe from gravity leaking to extra dimensions, Phys. Rev. D 65 (2002) 044023 [astro-ph/0105068] [INSPIRE].

[15] V.A. Rubakov and M.E. Shaposhnikov, Do We Live Inside a Domain Wall?, Phys. Lett. 125B (1983) 136 [INSPIRE]. 
[16] V.A. Rubakov and M.E. Shaposhnikov, Extra Space-Time Dimensions: Towards a Solution to the Cosmological Constant Problem, Phys. Lett. 125B (1983) 139 [inSPIRE].

[17] N. Arkani-Hamed, S. Dimopoulos, N. Kaloper and R. Sundrum, A Small cosmological constant from a large extra dimension, Phys. Lett. B 480 (2000) 193 [hep-th/0001197] [INSPIRE].

[18] S. Kachru, M.B. Schulz and E. Silverstein, Selftuning flat domain walls in $5-D$ gravity and string theory, Phys. Rev. D 62 (2000) 045021 [hep-th/0001206] [InSPIRE].

[19] C. Csáki, J. Erlich, C. Grojean and T.J. Hollowood, General properties of the selftuning domain wall approach to the cosmological constant problem, Nucl. Phys. B 584 (2000) 359 [hep-th/0004133] [INSPIRE].

[20] C. Charmousis, E. Kiritsis and F. Nitti, Holographic self-tuning of the cosmological constant, JHEP 09 (2017) 031 [arXiv: 1704.05075] [INSPIRE].

[21] T. Faulkner and J. Polchinski, Semi-Holographic Fermi Liquids, JHEP 06 (2011) 012 [arXiv: 1001.5049] [INSPIRE].

[22] E. Kiritsis, Gravity and axions from a random UV QFT, EPJ Web Conf. 71 (2014) 00068 [arXiv: 1408.3541] [INSPIRE].

[23] G.R. Dvali, G. Gabadadze and M. Porrati, Metastable gravitons and infinite volume extra dimensions, Phys. Lett. B 484 (2000) 112 [hep-th/0002190] [INSPIRE].

[24] G.R. Dvali, G. Gabadadze and M. Porrati, $4-D$ gravity on a brane in $5-D$ Minkowski space, Phys. Lett. B 485 (2000) 208 [hep-th/0005016] [INSPIRE].

[25] S.S. Gubser, Curvature singularities: The Good, the bad and the naked, Adv. Theor. Math. Phys. 4 (2000) 679 [hep-th/0002160] [INSPIRE].

[26] U. Gürsoy and E. Kiritsis, Exploring improved holographic theories for QCD: Part I, JHEP 02 (2008) 032 [arXiv:0707.1324] [inSPIRE].

[27] U. Gürsoy, E. Kiritsis and F. Nitti, Exploring improved holographic theories for QCD: Part II, JHEP 02 (2008) 019 [arXiv:0707.1349] [INSPIRE].

[28] S.S. Gubser and A. Nellore, Mimicking the QCD equation of state with a dual black hole, Phys. Rev. D 78 (2008) 086007 [arXiv:0804.0434] [InSPIRE].

[29] B. Gouteraux and E. Kiritsis, Generalized Holographic Quantum Criticality at Finite Density, JHEP 12 (2011) 036 [arXiv:1107.2116] [INSPIRE].

[30] B. Gouteraux, J. Smolic, M. Smolic, K. Skenderis and M. Taylor, Holography for Einstein-Maxwell-dilaton theories from generalized dimensional reduction, JHEP 01 (2012) 089 [arXiv: 1110.2320] [INSPIRE].

[31] I. Papadimitriou and K. Skenderis, Correlation functions in holographic RG flows, JHEP 10 (2004) 075 [hep-th/0407071] [INSPIRE].

[32] A.B. Clark, D.Z. Freedman, A. Karch and M. Schnabl, Dual of the Janus solution: An interface conformal field theory, Phys. Rev. D 71 (2005) 066003 [hep-th/0407073] [INSPIRE].

[33] J.K. Ghosh, E. Kiritsis, F. Nitti and L.T. Witkowski, Holographic RG flows on curved manifolds and quantum phase transitions, JHEP 05 (2018) 034 [arXiv:1711.08462] [INSPIRE].

[34] N. Kaloper, Bent domain walls as brane worlds, Phys. Rev. D 60 (1999) 123506 [hep-th/9905210] [INSPIRE]. 
[35] A. Karch and L. Randall, Locally localized gravity, JHEP 05 (2001) 008 [hep-th/0011156] [INSPIRE].

[36] A. Padilla, Cosmic acceleration from asymmetric branes, Class. Quant. Grav. 22 (2005) 681 [hep-th/0406157] [INSPIRE].

[37] A. Padilla, Infra-red modification of gravity from asymmetric branes, Class. Quant. Grav. 22 (2005) 1087 [hep-th/0410033] [INSPIRE].

[38] L. Gergely and R. Maartens, Asymmetric brane-worlds with induced gravity, Phys. Rev. D 71 (2005) 024032 [gr-qc/0411097] [INSPIRE].

[39] K. Koyama and K. Koyama, Brane induced gravity from asymmetric warped compactification, Phys. Rev. D 72 (2005) 043511 [hep-th/0501232] [INSPIRE].

[40] C. Charmousis, R. Gregory and A. Padilla, Stealth Acceleration and Modified Gravity, JCAP 10 (2007) 006 [arXiv:0706.0857] [InSPIRE].

[41] S. Banerjee, U. Danielsson, G. Dibitetto, S. Giri and M. Schillo, Emergent de Sitter cosmology from decaying AdS, arXiv:1807.01570 [INSPIRE].

[42] K. Koyama, A. Padilla and F.P. Silva, Ghosts in asymmetric brane gravity and the decoupled stealth limit, JHEP 03 (2009) 134 [arXiv:0901.0713] [INSPIRE].

[43] H.L. Verlinde, Holography and compactification, Nucl. Phys. B 580 (2000) 264 [hep-th/9906182] [INSPIRE].

[44] U.H. Danielsson and T. Van Riet, What if string theory has no de Sitter vacua?, Int. J. Mod. Phys. D 27 (2018) 1830007 [arXiv: 1804.01120] [INSPIRE].

[45] D. Andriot, On the de Sitter swampland criterion, Phys. Lett. B 785 (2018) 570 [arXiv: 1806.10999] [INSPIRE].

[46] G. Obied, H. Ooguri, L. Spodyneiko and C. Vafa, de Sitter Space and the Swampland, arXiv: 1806.08362 [INSPIRE].

[47] A. Amariti, C. Charmousis, D. Forcella, E. Kiritsis and F. Nitti, to appear.

[48] A. Kehagias and E. Kiritsis, Mirage cosmology, JHEP 11 (1999) 022 [hep-th/9910174] [INSPIRE].

[49] E. Kiritsis, Mirage cosmology and universe-brane stabilization, PoS (tmr99) 025.

[50] E. Kiritsis, F. Nitti and L. Silva Pimenta, Exotic RG Flows from Holography, Fortsch. Phys. 65 (2017) 1600120 [arXiv: 1611.05493] [INSPIRE].

[51] I. Papadimitriou and K. Skenderis, AdS/CFT correspondence and geometry, IRMA Lect. Math. Theor. Phys. 8 (2005) 73 [hep-th/0404176] [InSPIRE].

[52] I. Papadimitriou, Multi-Trace Deformations in AdS/CFT: Exploring the Vacuum Structure of the Deformed CFT, JHEP 05 (2007) 075 [hep-th/0703152] [INSPIRE].

[53] J. Lindgren, I. Papadimitriou, A. Taliotis and J. Vanhoof, Holographic Hall conductivities from dyonic backgrounds, JHEP 07 (2015) 094 [arXiv: 1505.04131] [INSPIRE].

[54] M.M. Caldarelli, A. Christodoulou, I. Papadimitriou and K. Skenderis, Phases of planar AdS black holes with axionic charge, JHEP 04 (2017) 001 [arXiv: 1612.07214] [INSPIRE].

[55] F. Nitti, L. Silva Pimenta and D.A. Steer, On multi-field flows in gravity and holography, JHEP 07 (2018) 022 [arXiv:1711.10969] [INSPIRE]. 
[56] U. Gürsoy, E. Kiritsis, F. Nitti and L. Silva Pimenta, Exotic holographic RG flows at finite temperature, JHEP 10 (2018) 173 [arXiv:1805.01769] [INSPIRE].

[57] C. Vafa, The String landscape and the swampland, hep-th/0509212 [INSPIRE].

[58] H. Ooguri and C. Vafa, On the Geometry of the String Landscape and the Swampland, Nucl. Phys. B 766 (2007) 21 [hep-th/0605264] [INSPIRE].

[59] D. Klaewer and E. Palti, Super-Planckian Spatial Field Variations and Quantum Gravity, JHEP 01 (2017) 088 [arXiv:1610.00010] [INSPIRE].

[60] E. Palti, The Weak Gravity Conjecture and Scalar Fields, JHEP 08 (2017) 034 [arXiv: 1705.04328] [INSPIRE].

[61] A. Hebecker, P. Henkenjohann and L.T. Witkowski, Flat Monodromies and a Moduli Space Size Conjecture, JHEP 12 (2017) 033 [arXiv: 1708. 06761] [INSPIRE].

[62] T.W. Grimm, E. Palti and I. Valenzuela, Infinite Distances in Field Space and Massless Towers of States, JHEP 08 (2018) 143 [arXiv: 1802.08264] [INSPIRE]. 